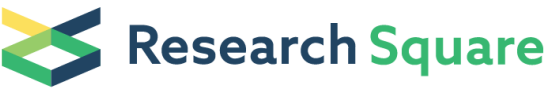 \\ Preprints are preliminary reports that have not undergone peer review. They should not be considered conclusive, used to inform clinical practice, or referenced by the media as validated information.
}

\section{Nanoscale Cutting Using Self-Excited Microcantilever}

\section{RUI YANG ( $\nabla$ yangrui2471@gmail.com )}

University of Tsukuba Graduate School Systems and Information Engineering: Tsukuba Daigaku Daigakuin System Joho Kogaku Kenkyuka https://orcid.org/0000-0002-5287-918X

\section{Ichiro Ogura}

National Institute of Advanced Industrial Science and Technology Advanced Manufacturing Research Institute: Sangyo Gijutsu Sogo Kenkyujo Seizo Gijutsu Kenkyu Bumon

\section{ZhenYan Jiang}

University of Tsukuba Graduate School Systems and Information Engineering: Tsukuba Daigaku Daigakuin System Joho Kogaku Kenkyuka

\section{LinJun An}

University of Tsukuba Graduate School Systems and Information Engineering: Tsukuba Daigaku Daigakuin System Joho Kogaku Kenkyuka

\section{Kiwamu Ashida}

National Institute of Advanced Industrial Science and Technology Advanced Manufacturing Research Institute: Sangyo Gijutsu Sogo Kenkyujo Seizo Gijutsu Kenkyu Bumon

\section{Hiroshi Yabuno}

University of Tsukuba Graduate School Systems and Information Engineering: Tsukuba Daigaku Daigakuin System Joho Kogaku Kenkyuka

\section{Research Article}

Keywords: Nanoscale cutting, AFM, Microcantilever, Self-excitation, Phase modulation

Posted Date: May 18th, 2021

DOl: https://doi.org/10.21203/rs.3.rs-482726/v1

License: (c) (1) This work is licensed under a Creative Commons Attribution 4.0 International License. Read Full License

Version of Record: A version of this preprint was published at Scientific Reports on January 12th, 2022. See the published version at https://doi.org/10.1038/s41598-021-04085-y. 


\title{
Nanoscale cutting using self-excited microcantilever
}

\author{
R. Yang · I. Ogura $\cdot$ Z.Y. Jiang · L.J. An · K. Ashida $\cdot$ H. Yabuno
}

Received: date / Accepted: date

\begin{abstract}
The application of self-excitation is proposed to improve the efficiency of the nanoscale cutting procedure based on use of a microcantilever in atomic force microscopy. The microcantilever shape is redesigned so that it can be used to produce vibration amplitudes with sufficient magnitudes to enable the excitation force applied by an actuator to be transferred efficiently to the tip of the microcantilever for the cutting process. A diamond abrasive that is set on the tip is also fabricated using a focused ion beam technique to improve the cutting effect. The natural frequency of the microcantilever is modulated based on the pressing load. Under conventional external excitation conditions, to maintain the microcantilever in its resonant state, it is necessary to vary the excitation frequency in accordance with the modulation. In this study, rather than using external excitation, the self-excitation cutting method is proposed to overcome this difficulty. The self-excited oscillation is produced by appropriate setting of the phase difference between the deflection signal of the microcantilever and the feedback signal for the actuator. In addition, it is demonstrated experimentally that the change in the phase difference enables us to control the amplitude of the self-excitation. As a result, control of the groove cutting depth is achieved via changes in the phase difference.
\end{abstract}

Keywords Nanoscale cutting - AFM - Microcantilever · Self-excitation $\cdot$ Phase modulation

R. Yang $\cdot$ Z.Y. Jiang $\cdot$ L.J. An $\cdot$ H. Yabuno

Graduate School of Systems and Information Engineering, University

of Tsukuba, 1-1-1, Tennodai Tsukuba City, 305-8573, Japan

E-mail: yabuno@esys.tsukuba.ac.jp

I. Ogura $\cdot$ K. Ashida

Advanced Manufacturing Research Institute, National Institute of Advanced Industrial Science and Technology, 1-2-1, Namiki, Tsukuba, 305-0044, Japan

\section{Introduction}

Various types of micromachining technology have been established for use in microelectromechanical systems (MEMS) manufacture [1]. Wet and dry etching processes [2,3], which enable removal of materials for silicon bulk micromachining, are excessively dependent on variations in the concentrations of the rare chemicals used and the extreme environmental created $[4,5]$. Laser processing [6] and focused ion beam processing [7] techniques, which ablate materials using focused beams of light or ions, respectively, is limited in processing accuracy by the divergence of the beam focal point described by the wave theory of light [8] and the alteration in the target layer due to heating by the incident beam [9]. Also, these micromachining technologies are unsuitable for nanoscale precision fabrication of structures with nonflat surfaces. However, this problem was previously solved using cutting methods that relied on a force control system that could cut inclined or curved surfaces to the same depth [10-12].

Atomic force microscopy (AFM) [13], which has been used for nanoscale imaging of surfaces, has also been used as a force control system for nanomachining to cut groove of approximately $10 \mathrm{~nm}$ depth in single-crystal silicon [14]. The control of the distance between the microcantilever tip and the sample surface in the AFM system was appropriate for application of a constant force during the cutting process. In addition, a microcantilever has been equipped with a diamond on the tip, which acted as a useful cutting tool [15]. The required groove has been achieved to be cut to greater depths by increasing the pressing load set on the tip of the microcantilever [16]. Nanomachining technology using the microcantilever in the AFM system has been demonstrated to be a low-cost, high-precision method for cutting of various materials into nanodots, nanolines, and even two-dimensional and three-dimensional structures 
[17]. Those previous methods have limitations in improvement of the process efficiency. On the other hand, various types of vibration-assisted machining method have been proposed for enhancing process efficiency, where either external excitation or self-excitation is generally used $[18,19]$. This study intends to propose a new practical nanomachining technology and discuss the theoretic support by the fusion of the vibration-assistance.

In this research, the shape of the microcantilever is redesigned by adding a base step to produce the required magnitude of the vibration amplitude. A diamond abrasive is set on the tip and fabricated sharper using focused ion beam (FIB) technology. Because the natural frequency of the microcantilever is modulated based on the different pressing loads applied, the microcantilever cannot be maintained in the resonant state at a constant excitation frequency under external excitation. To overcome this difficulty, a new vibrational cutting method is proposed that uses a self-excited microcantilever. Through appropriate setting of the phase difference between the microcantilever deflection signal and the feedback signal for the actuator, self-excited oscillation in the microcantilever is produced experimentally and the resonance mechanism is then clarified theoretically. Furthermore, it is demonstrated experimentally that variation of the phase difference enables control of the amplitude of the selfexcitation. As a result, variable groove depths are cut by changing the phase difference appropriately.

\section{Self-excitation cutting method using AFM system}

\subsection{AFM application to nanoscale cutting}

Figure 1 shows the AFM system used for the nanoscale cutting operations. The microcantilever is connected to the bottom of the piezo actuator through the holder. The piezo actuator is then charged to cause the microcantilever to oscillate. The optical angle sensor, which consists of a laser and a quadrant photodiode, measures the deflection of the microcantilever tip. The tube scanner is charged to move the workpiece within the $x-y$ plane and also performs positional control in the $z$-direction to maintain a constant pressing load during cutting.

\subsection{Redesigned microcantilever and diamond abrasive}

By setting the same pressing load on the microcantilever tip, the grooves were cut to the same depth regardless of the application of the excitation because the response amplitude at the microcantilever tip was too small, even in the resonant state. Therefore, to produce a sufficient response amplitude magnitude to ensure that the excitation force can be transferred efficiently to the tip, the microcantilever shape is re-

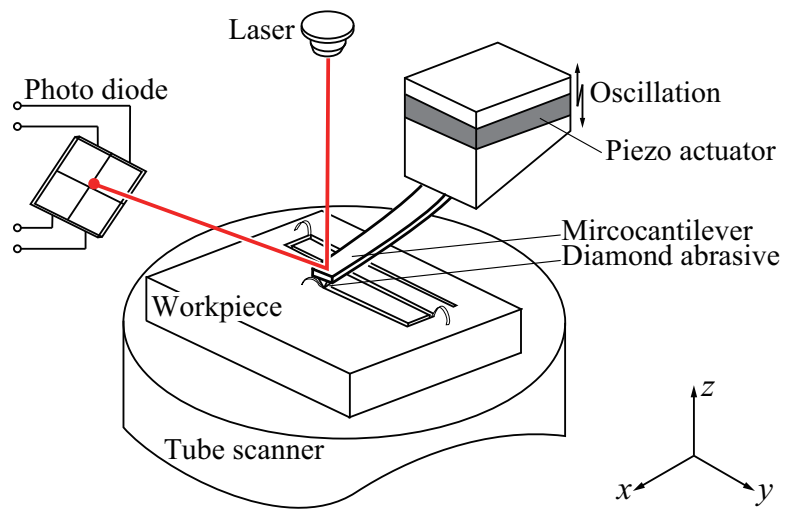

Fig. 1 Schematic diagram of AFM system applied to nanoscale vibration cutting method. The system includes a workpiece, a microcantilever with a diamond set on its tip, a piezo actuator to enable the microcantilever oscillation, an optical angle sensor consisting of a laser and a quadrant photodiode to measure the deflection of the microcantilever tip, a tube scanner to move the workpiece in the $x-y$ plane and vary the distance between the microcantilever and the workpiece in the $z$-direction.

(a)

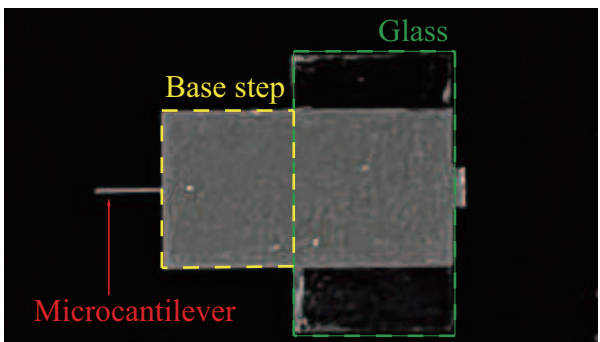

(b)

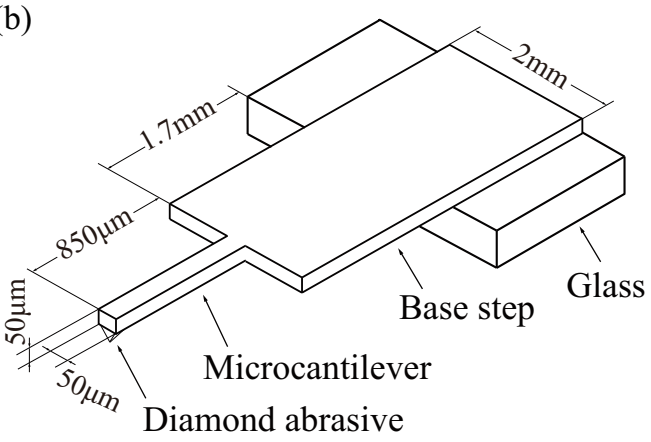

Fig. 2 Photo (a) and Schematic diagram (b) of redesigned microcantilever. The length, width and thickness of the base step are $1.7 \mathrm{~mm}$, $2 \mathrm{~mm}$ and $50 \mu \mathrm{m}$, respectively. The length, width and thickness of the microcantilever are $850 \mu \mathrm{m}, 50 \mu \mathrm{m}$ and $50 \mu \mathrm{m}$, respectively.

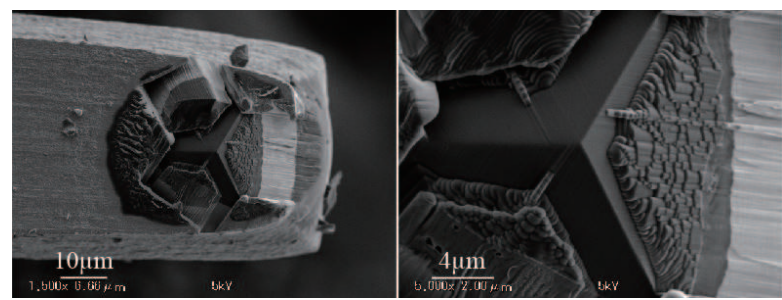

Fig. 3 Diamond abrasive. This abrasive is set on the microcantilever tip and fabricated using focused ion beam technique. The angle of the fabricated diamond is 90 degrees; this angle is formed by the sides and the triangular faces at the profile vertex. 
designed as shown in Fig. 2(a). In this design, a base step is connected to the microcantilever. The length, width and thickness of this base step are $1.7 \mathrm{~mm}, 2 \mathrm{~mm}$ and $50 \mu \mathrm{m}$, respectively. The length, width and thickness of the microcantilever are $850 \mu \mathrm{m}, 50 \mu \mathrm{m}$ and $50 \mu \mathrm{m}$, respectively, as shown in Fig. 2(b).

A diamond has previously been fabricated using the FIB technique to achieve an improved cutting effect [20]. For this reason, we set a diamond abrasive on the microcantilever tip and fabricated it into a tetrahedron shape using the FIB technique. The angle of the diamond is 90 degrees and this angle is formed by the sides and the triangular faces at the profile vertex, as shown in Fig. 3. Hereinafter, this redesigned microcantilever is referred to as the microcantilever.

\subsection{Natural frequency of microcantilever}

The first natural frequency of the cantilever is calculated [21] to be

$f_{1}=\frac{\lambda_{1}{ }^{2}}{2 \pi l^{2}} \sqrt{\frac{E I}{\rho A}}$,

where $\lambda_{1}, l, \rho$ and $A$ denote the first-order eigenvalue, the length, the density and the cross-sectional area of the microcantilever, respectively. $E$ and $I$ are the Young's modulus and the moment of inertia of the area, respectively, as summarized in Table 1. Using Eq. (1), the first natural frequencies of the microcantilever and the base step are calculated to be $55.97 \mathrm{kHz}$ and $14.06 \mathrm{kHz}$, respectively.

Table 1 Parameters of the microcantilever.

\begin{tabular}{llll}
\hline Description & Symbol & Value & Unit \\
\hline First-order eigenvalue & $\lambda_{1}$ & 1.875 & \\
Young's modulus & $E$ & $199.14 \times 10^{3}$ & $\mathrm{~Pa}$ \\
$\begin{array}{l}\text { Density } \\
\text { Length of microcantilever }\end{array}$ & $l_{m}$ & $0.85 \times 10^{-3}$ & $\mathrm{~kg} / \mathrm{m}^{3}$ \\
$\begin{array}{l}\text { Length of base step } \\
\text { Moment of inertia of }\end{array}$ & $l_{b}$ & $1.7 \times 10^{-3}$ & $\mathrm{~m}$ \\
$\begin{array}{l}\text { area of microcantilever } \\
\text { Moment of inertia of }\end{array}$ & $I_{m}$ & $0.52 \times 10^{-18}$ & $\mathrm{~m}^{4}$ \\
$\begin{array}{l}\text { area of base step } \\
\begin{array}{l}\text { Cross-sectional area } \\
\text { of microcantilever }\end{array}\end{array}$ & $A_{m}$ & $0.25 \times 10^{-16}$ & $\mathrm{~m}^{4}$ \\
$\begin{array}{l}\text { Cross-sectional area } \\
\text { of base step }\end{array}$ & $A_{b}$ & $0.1 \times 10^{-6}$ & $\mathrm{~m}^{2}$ \\
\hline
\end{tabular}

Figure 4(a) shows the frequency response curve under external excitation in air. The first natural frequencies of the microcantilever and the base step were measured to be 44.35 $\mathrm{kHz}$ and $12.88 \mathrm{kHz}$, respectively. These values show close agreement with the theoretical frequencies. Figure 4(b) shows that the first natural frequency of the microcantilever is 15.49 $\mathrm{kHz}$ under a pressing load of $400 \mu \mathrm{N}$. The response amplitude is of sufficient magnitude to transfer the excitation force to the tip effectively. Furthermore, because the boundary condition of the microcantilever tip is changed under application of the pressing load, the response amplitude disappears in the case where the excitation frequency is within the vicinity of the natural frequency of the microcantilever, but the response amplitude is maintained in the case where the excitation frequency is in the vicinity of the natural frequency of the base step. Only one remaining vibration mode is self-excited according to the method proposed in the following section.
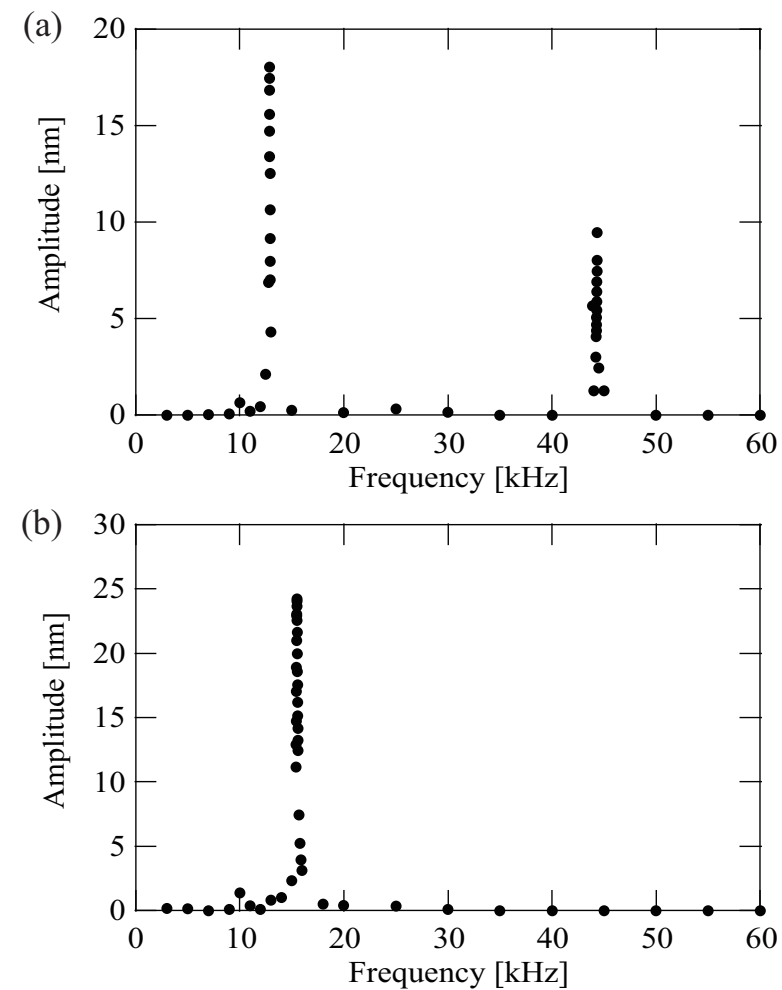

Fig. 4 Response frequency curve (a) in air and (b) under a $400 \mu \mathrm{N}$ pressing load. The first natural frequencies of the microcantilever and the base step in air were experimentally measured to be $44.35 \mathrm{kHz}$ and $12.88 \mathrm{kHz}$, respectively. The first natural frequency under a $400 \mu \mathrm{N}$ pressing load was experimentally measured to be $15.49 \mathrm{kHz}$.

\section{Cutting method using self-excited microcantilever}

3.1 Change in natural frequency under various pressing loads

In a series of experiments performed using the external excitation cutting method, it is determined experimentally that 
the natural frequency of the microcantilever changes under the application of different pressing loads, as shown in Fig. 5. Therefore, under the condition where the pressing load is changed, the microcantilever is not maintained in its resonant state under application of the external excitation.

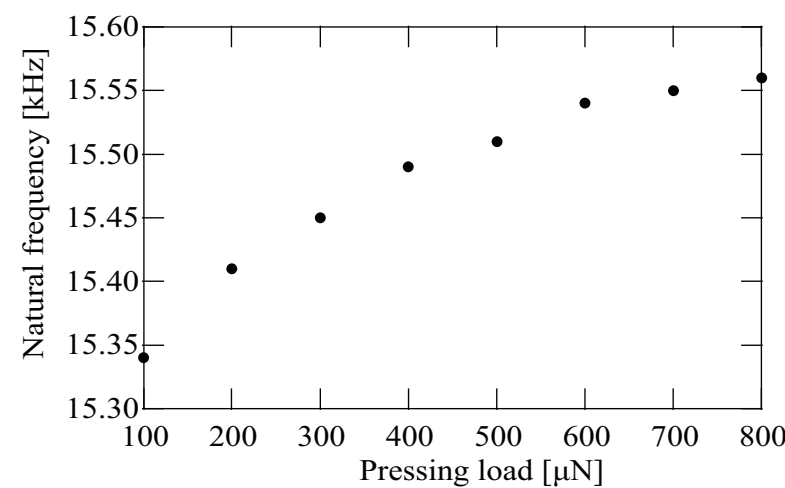

Fig. 5 Natural frequency of microcantilever under various pressing loads with application of external excitation.

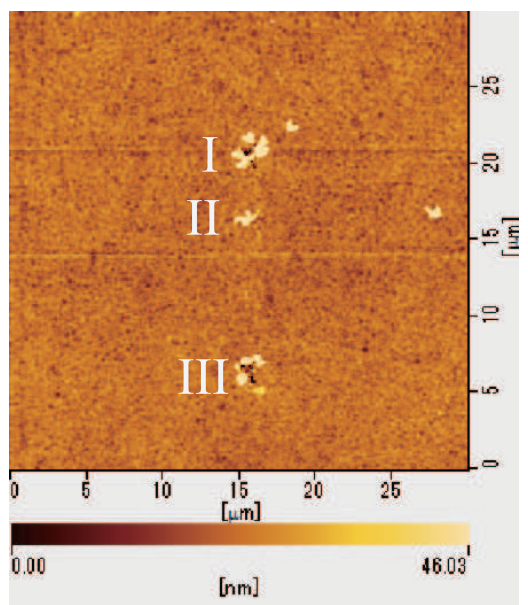

Fig. 6 Image of grooves cut with the external excitation cutting method acquired using AFM. The upper groove is I, the middle groove is II and the lower groove is III.

Figure 6 shows three grooves that were cut using the external excitation cutting method; the image shows the experimental results measured by AFM. Figure 7 shows the sectional views of these grooves. The upper groove (I) and the middle one (II) in Fig. 6 were cut under pressing loads of $200 \mu \mathrm{N}$ and $400 \mu \mathrm{N}$, respectively, at an excitation frequency of $15.41 \mathrm{kHz}$, which is the natural frequency under the pressing load of $200 \mu \mathrm{N}$. The lower groove (III) in Fig. 6 was cut under application of a $400 \mu \mathrm{N}$ pressing load at an excitation frequency of $15.49 \mathrm{kHz}$, which is the natural frequency under the pressing load of $400 \mu \mathrm{N}$. The results are summarized in Table 2. As indicated by the sectional view shown in Fig. 7(b), the middle one (II) could not be cut into groove.
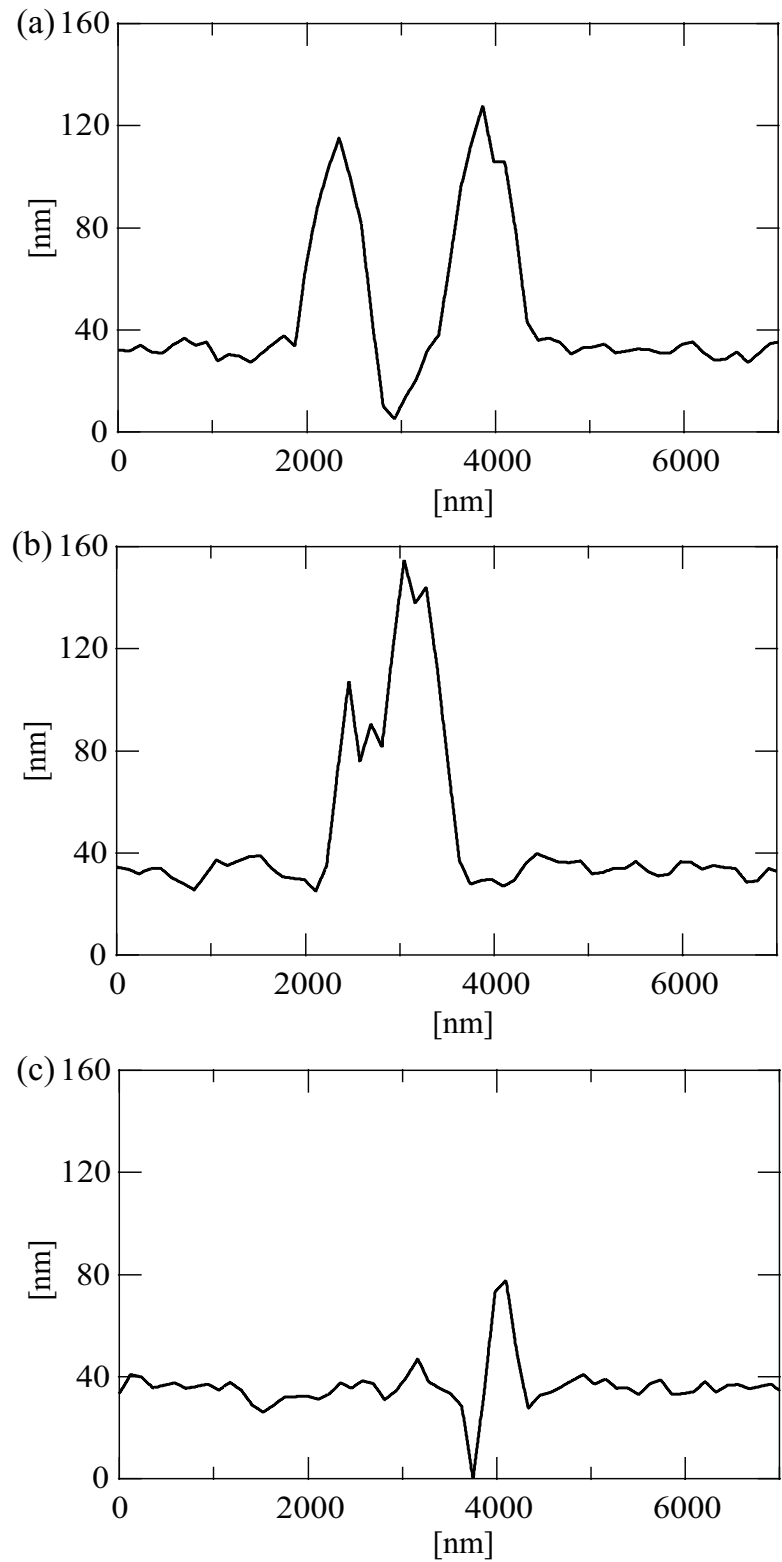

Fig. 7 Sectional views of three cut grooves acquired using AFM. (a), (b) and (c) represent the sectional views of the three cut grooves I, II and III, respectively.

Table 2 Results of groove cutting using the external excitation cutting method.

\begin{tabular}{lllll}
\hline $\begin{array}{l}\text { Cutting } \\
\text { groove }\end{array}$ & $\begin{array}{l}\text { Pressing } \\
\text { load } \\
{[\mu \mathrm{m}]}\end{array}$ & $\begin{array}{l}\text { Excitation } \\
\text { frequency } \\
{[\mathrm{kHz}]}\end{array}$ & $\begin{array}{l}\text { Amplitude } \\
{[\mathrm{nm}]}\end{array}$ & $\begin{array}{l}\text { Depth } \\
{[\mathrm{nm}]}\end{array}$ \\
\hline I & 200 & 15.41 & 25.51 & 30.61 \\
II & 400 & 15.41 & 11.64 & Null \\
III & 400 & 15.49 & 32.08 & 35.27 \\
\hline
\end{tabular}


Comparison of the upper groove (I) with the middle groove (II) shows that the groove cannot be cut to be deeper when the microcantilever is not in its resonant state. Comparison of the upper (I) and lower grooves (III) reveals that a deeper groove can be cut if the microcantilever remains in the resonant state, even if the natural frequency is changed under the larger pressing load. Consequently, in the case of vibrational cutting using external excitation, it is necessary to measure the natural frequency modulation depending on each pressing load in advance to tune the excitation frequency to be the natural frequency during the process. To overcome this difficulty, a self-excitation cutting method is proposed in this research that can maintain the microcantilever in its resonant state independently of the pressing load during cutting without the need for tuning the excitation frequency.

\subsection{Analytical model of self-excited microcantilever}

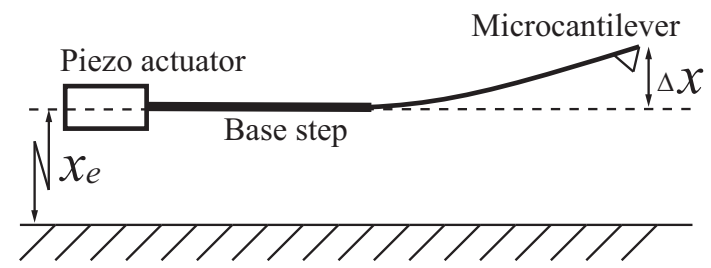

Fig. 8 Schematic diagram of self-excited microcantilever.

Self-excitation of the microcantilever used in AFM has been produced via linear feedback control [22,23]. We therefore introduce a discretized model with a single-degree-offreedom for the microcantilever system with the base step, as shown in Fig. 8. The dynamics of the proposed self-excited microcantilever in air are governed by

$m\left(\ddot{x}_{e}+\Delta \ddot{x}\right)+c \Delta \dot{x}+k \Delta x=0$,

where $m, c$ and $k$ are the mass, the damping and the stiffness of the microcantilever, respectively. $\Delta x$ represents the displacement of microcantilever tip. $x_{e}$ is the applied displacement for self-excitation. We set the integral feedback to be

$x_{e}=-k_{\text {lin }} \int \Delta x d t$,

where $k_{l i n}$ is the linear feedback gain. Substitution of Eq. (3) into Eq. (2) yields

$m \Delta \ddot{x}+\left(c-c_{\text {lin }}\right) \Delta \dot{x}+k \Delta x=0$,

where $c_{l i n}=m k_{l i n}$. We produce the self-excited oscillation using negative damping. Therefore, the feedback gain $c_{\text {lin }}$ is set to ensure that $c-c_{\text {lin }}$ is negative. In practice, the equivalent beam displacement $\Delta x$ is measured using an optical angle sensor and the displacement $x_{e}$ is applied by a piezo actuator, as shown in Fig. 9(a). The input signal to the piezo actuator is generated by the feedback circuit, as shown in Fig. 9(b), where the input and output are the output signal from the optical angle sensor and the input signal to the piezo actuator, respectively. Hereinafter, this circuit is called the self-excited electronic circuit.

(a)

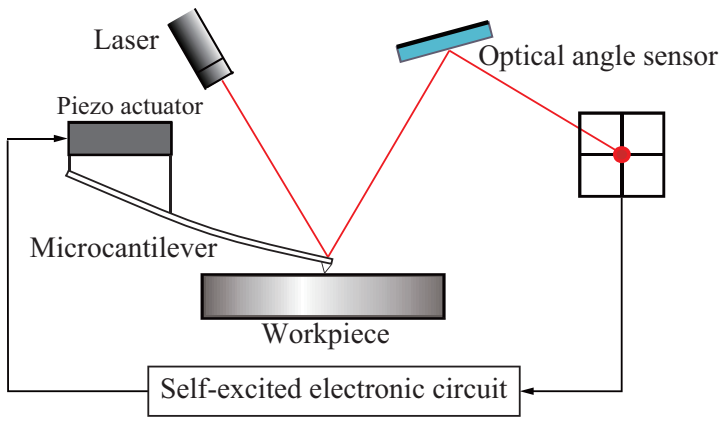

(b) Voltage follower Phase shifter Inverted amplifter (I)
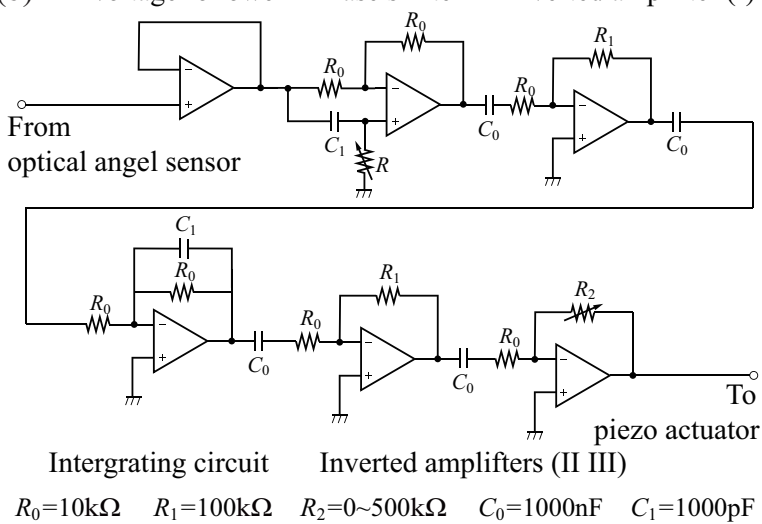

Fig. 9 Schematic diagram of the self-excited system. (a) includes a workpiece, a microcantilever, a piezo actuator, a self-excited electronic circuit and an optical angle sensor that consists of a laser and a photodiode. (b) includes a voltage follower, a phase shifter, an integrating circuit and three inverting amplifiers.

3.3 Comparison between vibrational cutting methods using external excitation and self-excitation

We now compare the two types of vibrational cutting method using external excitation and self-excitation. In the external excitation cutting method, the microcantilever is excited at its natural frequency measured under a $400 \mu \mathrm{N}$ pressing load with a $20 \mathrm{Vp}-\mathrm{p}$ sinusoidal signal. In the self-excitation cutting method, the input of the piezo actuator is adjusted to be $20 \mathrm{Vp}$-p via linear feedback control. The depths of the grooves cut under each pressing load are shown in Fig. 10, 
where the triangles and the dots represent the cutting depths achieved when using the external excitation cutting method and the self-excitation cutting method, respectively.

Because the self-excitation cutting method maintains the resonant state automatically under any pressing load, the grooves are cut more deeply under each pressing load than in the external excitation case. Therefore, it is demonstrated experimentally that the self-excitation cutting method offers higher efficiency for nanoscale cutting regardless of the pressing load.

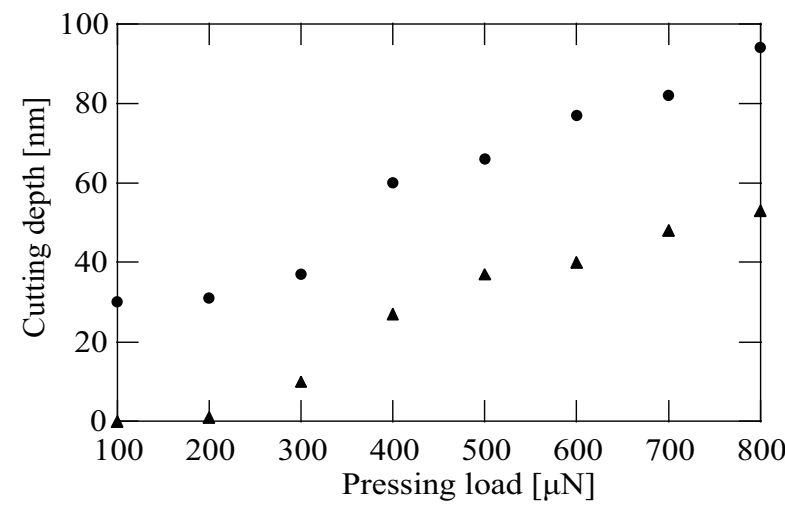

Fig. 10 Depths of grooves cut under each pressing load with two types of cutting method. The triangles and the dots represent the depths using the external excitation cutting method and the self-excitation cutting method, respectively.

\section{Change in cutting depth with the self-excitation cutting method}

\subsection{Amplitude change using linear feedback control}

To cut a groove with variable depth, amplitude control of the microcantilever is required. The solution to Eq. (4) can easily be expressed as

$\Delta x(t)=e^{\sigma t}\left(a_{1} \cos \omega_{d} t+a_{2} \sin \omega_{d} t\right)$.

where $\sigma=\left(c_{\text {lin }}-c\right) / 2 m$ and $\omega_{d}=\sqrt{4 m k-\left(c_{\text {lin }}-c\right)} / 2 m$. Here, $a_{1}$ and $a_{2}$ are constants that are determined by the initial conditions. The amplitude increases to infinity in theory in the case where the negative damping $\sigma>0$. However, in the experimental results, the response amplitude is kept constant. It is well known that the self-excited oscillator can only maintain a constant amplitude when external nonlinear effects act on the system [24]. We do consider methods to change the amplitude experimentally, but the theoretical investigation required to determine the source of the nonlinearity for the constant amplitude will form part of a future work.
Figure 11 shows the change in the response amplitude under application of a $400 \mu \mathrm{N}$ pressing load caused by variation of the feedback gain. In the case where the linear feedback gain $k_{\text {lin }}$ is above approximately $160[1 / \mathrm{s}]$, the selfexcited oscillation is produced. Even if the linear feedback gain is increased, the response amplitude does not change greatly. Variation of the feedback gain cannot be used for the amplitude control required to change the depth of the cut groove. Therefore, another way must be found to change the magnitude of the amplitude.

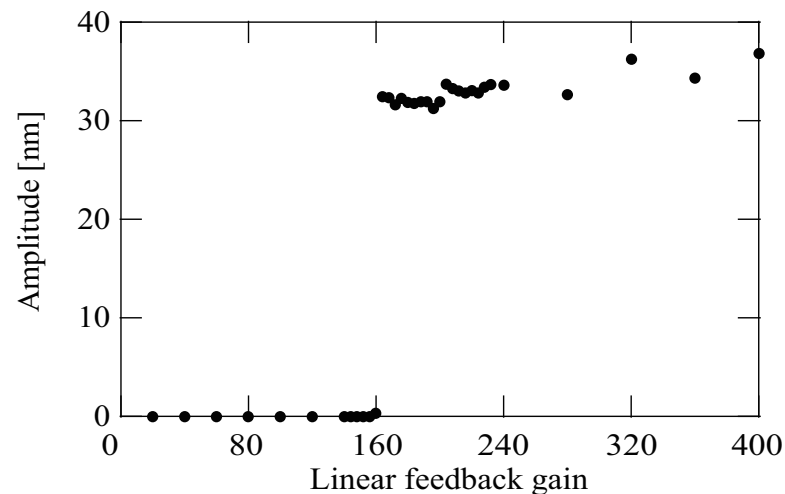

Fig. 11 Change in amplitude caused by controlling linear feedback gain $k_{\text {lin }}[1 / \mathrm{s}]$ under application of a $400 \mu \mathrm{N}$ pressing load.

4.2 Theoretical analysis of self-excitation using phase difference

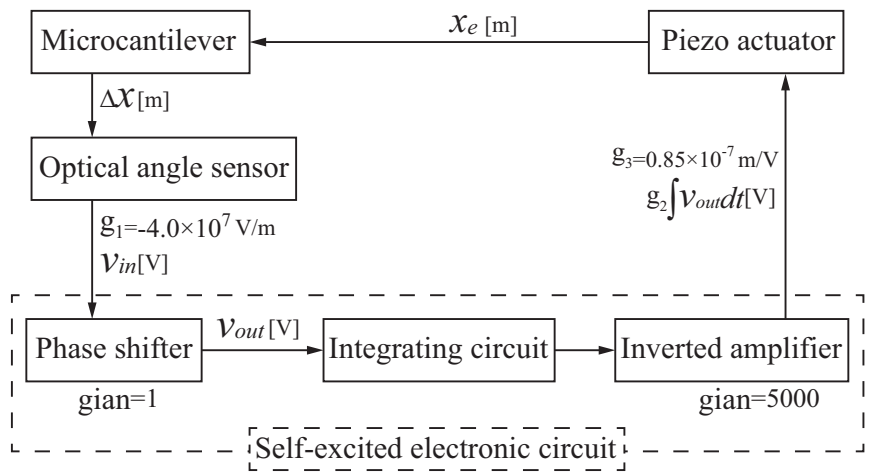

Fig. 12 Block diagram of the self-excited system.

A method based on linear feedback control was proposed in section 4.1; however, this method is not suitable for amplitude control. In this section, we theoretically consider another method to produce the self-excitation that involves setting the linear feedback for negative damping and shifting 
the phase difference between the microcantilever deflection signal and the feedback signal for amplitude control. The experimental verification of this method is discussed in the next section.

Figure 12 shows a diagram of the entire self-excited vibration system. To perform a theoretical analysis of the process of self-excitation by phase modulation, we rewrite $\Delta x$ and $x_{e}$ as the input and output voltages of the phase shifter $v_{\text {in }}$ and $v_{\text {out }}$ using the relationships $v_{\text {in }}=g_{1} \Delta x$ and $x_{e}=$ $g_{2} g_{3} \int v_{\text {out }} d t$, respectively, where $g_{1}$ is the sensitivity of the optical angle sensor, $g_{2}(5000[1 / \mathrm{s}])$ is the product of the gains of the inverted amplifier (I), the integration circuit and the inverted amplifiers (II) (III), which are 10, 1/s, 10 and 50 , respectively, $g_{3}\left(0.85 \times 10^{-7} \mathrm{~m} / \mathrm{V}\right)$ is the piezoelectric coefficient of the piezo actuator that is experimentally determined subsequently in accordance with the experimental value. Consequently, the equation for the self-excitation of the microcantilever and the differential equation for the phase shifter in the self-excited electronic circuit are rewritten as

$$
\left\{\begin{array}{l}
m \ddot{v}_{\text {in }}+c \dot{v}_{\text {in }}+k v_{\text {in }}=-g_{1} g_{2} g_{3} m \dot{v}_{\text {out }}, \\
C_{1} R \dot{v}_{\text {in }}-v_{\text {in }}=C_{1} R \dot{v}_{\text {out }}+v_{\text {out }},
\end{array}\right.
$$

where $C_{1}$ and $R$ are the values of the capacitance and the resistance in the phase shifter, respectively, as shown in Fig. 9(b). Equation (6) is nondimensionalized using the dimensionless time $t^{*}=\sqrt{\mathrm{k} / \mathrm{m}} t$ as follows

$$
\left\{\begin{array}{l}
\ddot{v}_{\text {in }}+\gamma \dot{v}_{\text {in }}+v_{\text {in }}=-\beta \dot{v}_{\text {out }} \\
\alpha \dot{v}_{\text {in }}-v_{\text {in }}=\alpha \dot{v}_{\text {out }}+v_{\text {out }}
\end{array}\right.
$$

where

$$
\alpha=C_{1} R \sqrt{\frac{k}{m}}, \quad \beta=g_{1} g_{2} g_{3} \sqrt{\frac{m}{k}}, \quad \gamma=\frac{c}{\sqrt{m k}} .
$$

Equation (7) is then rewritten into matrix form as

$$
\frac{\mathrm{d}}{\mathrm{d} t^{*}}\left[\begin{array}{c}
v_{\text {in }} \\
\dot{v}_{\text {in }} \\
v_{\text {out }}
\end{array}\right]=A\left[\begin{array}{c}
v_{\text {in }} \\
\dot{v}_{\text {in }} \\
v_{\text {out }}
\end{array}\right]
$$

where

$A=\left[\begin{array}{ccc}0 & 1 & 0 \\ \frac{\beta}{\alpha}-1 & -\gamma-\beta & \frac{\beta}{\alpha} \\ -\frac{1}{\alpha} & 1 & -\frac{1}{\alpha}\end{array}\right]$.

Using eigenvalue analysis, we investigate the possibility that self-excitation can be produced by varying the dimensionless parameter $\alpha$, which is related to the value of the resistance $R$ in the phase shifter. The characteristic equation of the matrix in Eq. (10) is derived as

$$
\lambda^{3}+\left(\beta+\gamma+\frac{1}{\alpha}\right) \lambda^{2}+\left(1-\frac{\beta-\gamma}{\alpha}\right) \lambda+\frac{1}{\alpha}=0 .
$$

The system parameters are summarized in Table 3. The piezoelectric coefficient of the piezo actuator is identified experimentally using eigenvalue analysis.

Table 3 Parameters of the microcantilever and the self-excited system.

\begin{tabular}{llll}
\hline Description & Symbol & Value & Unit \\
\hline Mass & $m$ & $1.37 \times 10^{-6}$ & $\mathrm{~kg}$ \\
Stiffness & $k$ & 260 & $\mathrm{~N} / \mathrm{m}$ \\
Damping coefficient & $c$ & $0.12 \times 10^{-3}$ & $\mathrm{~Pa} \cdot \mathrm{s}$ \\
Capacitance & $C_{1}$ & $1.0 \times 10^{-9}$ & $\mathrm{~F}$ \\
Sensibility of & $g_{1}$ & $-4.0 \times 10^{7}$ & $\mathrm{~V} / \mathrm{m}$ \\
optical angle sensor & & & \\
\hline
\end{tabular}

To realize self-excitation in a third-order system, the eigenvalues must be one negative real root and a pair of complex conjugate roots with a positive real part. According to the results shown in Fig. 14, the self-excitation of the microcantilever in air begins when the resistance is set at $40.6 \mathrm{k} \Omega$. Using the first equation in Eq. (8), we obtain $\alpha_{c r}=0.5603$ at the critical point with a negative real eigenvalue $q$ and a pair of conjugate purely imaginary eigenvalues $\pm i \omega$. Substitution of $i \omega$ into Eq. (10) and separation of the real and imaginary parts yields

$\left\{\begin{array}{l}\operatorname{Re}:\left(\beta+\gamma+\frac{1}{\alpha_{\mathrm{cr}}}\right) \omega^{2}-\frac{1}{\alpha_{\mathrm{cr}}}=0, \\ \operatorname{Im}: \omega^{3}-\left(1-\frac{\beta-\gamma}{\alpha_{\mathrm{cr}}}\right) \omega=0 .\end{array}\right.$

From Eq. (12), the relationship between $\alpha_{c r}$ and $\beta$ is calculated to be

$\alpha_{c r} \beta^{2}+\left(1-\alpha_{c r}^{2}\right) \beta-\gamma\left(\alpha_{c r}^{2}+\alpha_{c r} \gamma+1\right)=0$.

We then obtain $\beta=-1.2366$. From the second equation in Eq. (8), the piezoelectric coefficient $g_{3}$ is identified experimentally as $0.85 \times 10^{-7} \mathrm{~m} / \mathrm{V}$, where the linear feedback $g_{2}$ is 5000 [1/s], in accordance with the experimental value.

Because the parameters for the entire self-excited system have been determined, the root locus produced by varying $\alpha$ is shown in Fig. 13, where we change $\alpha$ from 0 to $\infty$, which are represented by the symbols $\times$ and $\circ$, respectively. Meanwhile, the real part of a pair of complex conjugate roots can be changed from negative to positive but the real root remains negative. The required self-excitation can be produced by setting the resistance value $R$ appropriately. However, it is predicted using linear theory that the amplitude will grow infinitely. The amplitude remains constant, as shown in the subsequent experiments. Because selfexcitation with the steady-state amplitude is produced by the 
nonlinear damping term as a van der Pol oscillator[25], the cause may be dependent on the nonlinear characteristics of the contact force between the microcantilever and the workpiece. Further theoretical discussions will form part of a future work.

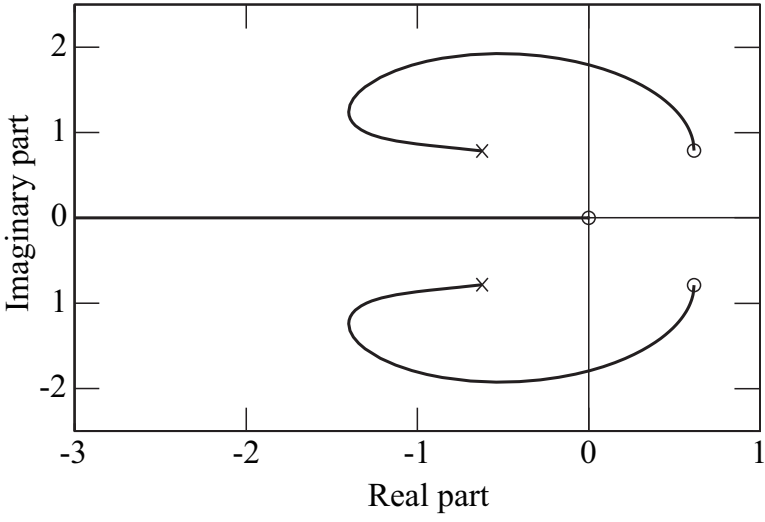

Fig. 13 Root locus of the self-excited system.

\subsection{Amplitude control using the phase modulation}

A method based on phase modulation is proposed for amplitude control of the self-excited microcantilever. In air, a constant linear feedback gain is set in advance. We then vary the resistance value $R$ in the phase shifter from $20 \mathrm{k} \Omega$ to 50 $\mathrm{k} \Omega$ to cause the phase difference between the input and the output of the self-excited electronic circuit to shift. When the resistance value is varied from $40.6 \mathrm{k} \Omega$ to $50 \mathrm{k} \Omega$, the response frequency is measured to change in the neighborhood of $13.03 \mathrm{kHz}$, which is almost agreement with the natural frequency of the base step $12.88 \mathrm{kHz}$, therefore we can conclude that only the base step is in the self-excited oscillation caused by appropriate setting of the phase difference, as shown in Fig. 14(a). In the same way, the microcantilever can be self-excited individually when the resistance value is varied from $23 \mathrm{k} \Omega$ to $40.4 \mathrm{k} \Omega$, as shown in Fig. 14(b), according to the response frequency measured to change in the neighborhood of $45.98 \mathrm{kHz}$, which is almost agreement with the natural frequency of the microcantilever $44.35 \mathrm{kHz}$.

Under application of a $400 \mu \mathrm{N}$ pressing load, we changed the resistance value in the phase shifter from $10 \mathrm{k} \Omega$ to 35 $\mathrm{k} \Omega$ with a constant linear feedback gain. Figure 15(a) shows that the change in the resistance value causes the phase difference to shift. Figure 15(b) shows the change in the amplitude magnitude caused by the phase shift. Above $14 \mathrm{k} \Omega$, self-excitation occurs with the single response frequency of $15.49 \mathrm{kHz}$, which is approximately equal to the natural frequency under the application of the $400 \mu \mathrm{N}$ pressing load, as
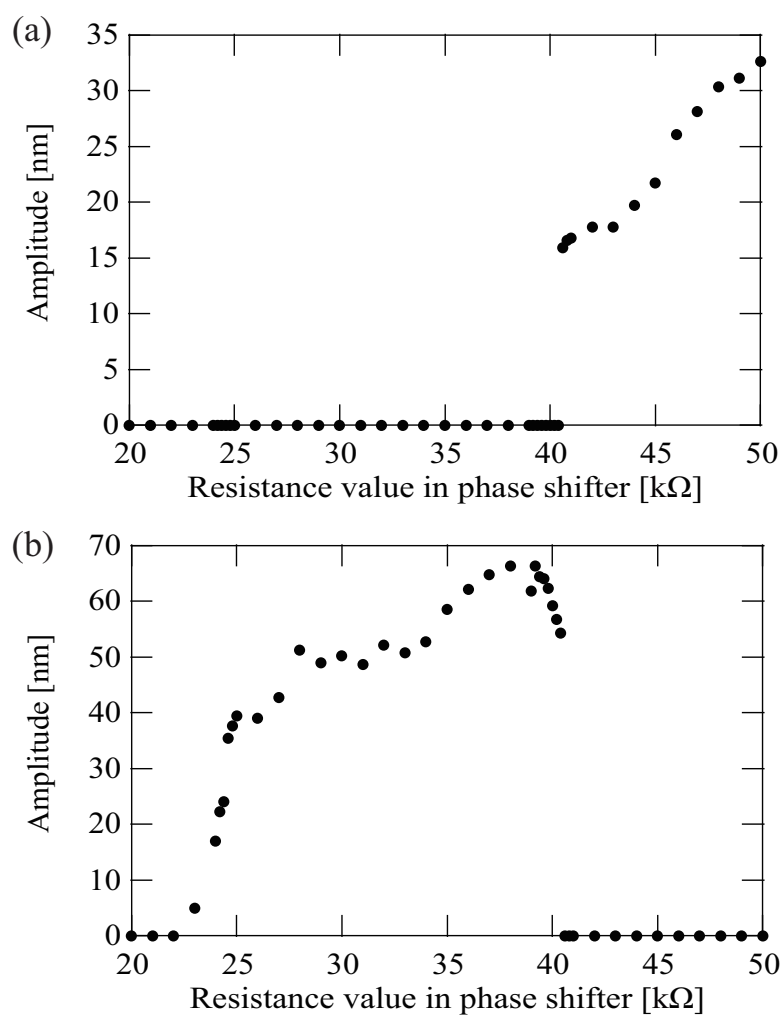

Fig. 14 Change in the amplitude magnitude in air caused by phase modulation. (a) and (b) show the amplitude magnitude changes in the cases when the base step and the microcantilever are self-excited, respectively, while the resistance value in the phase shifter is varied.
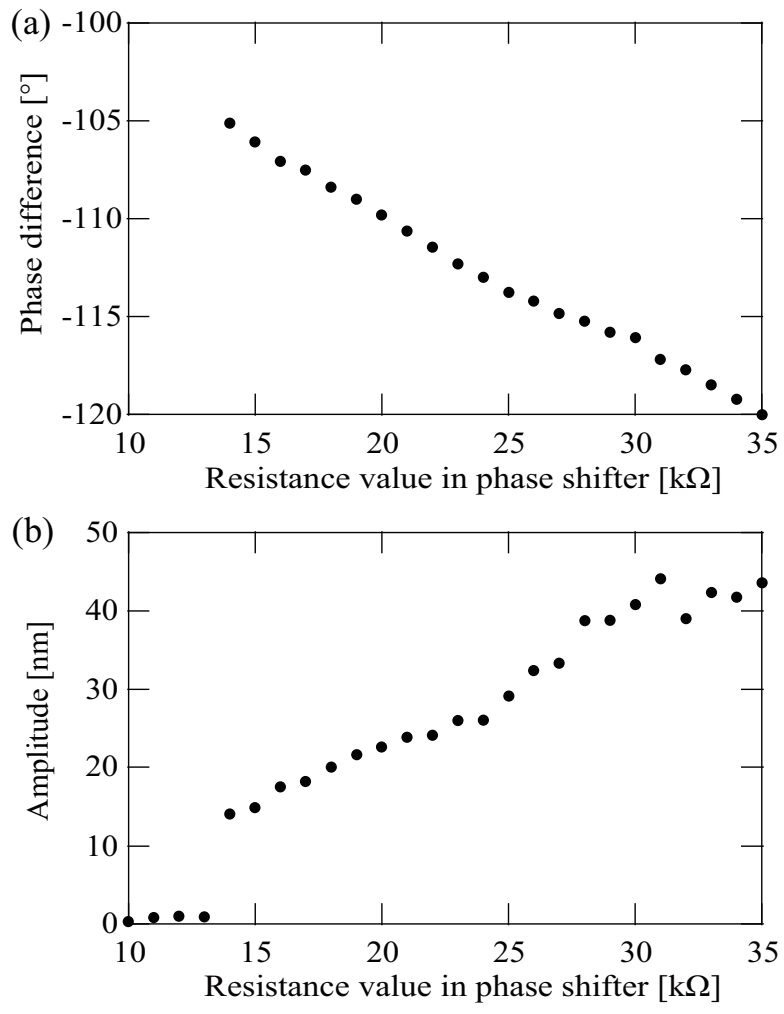

Fig. 15 Results for the phase difference and amplitude changes caused by phase modulation under application of a $400 \mu \mathrm{N}$ pressing load while the resistance value in the phase shifter was varied from $10 \mathrm{k} \Omega$ to 35 $\mathrm{k} \Omega$. (a) Change in the phase difference. (b) Change in the amplitude magnitude. 
shown in Fig. 4(b). By shifting the phase difference, the amplitude can be varied arbitrarily under the $400 \mu \mathrm{N}$ pressing load to realize different groove cutting depths, as discussed in section 4.4 .

4.4 Varying the cutting depth with a controlled amplitude using the phase modulation

In the cutting experiments performed under application of the $400 \mu \mathrm{N}$ pressing load, the four resistance values in the phase shifter were set as $15 \mathrm{k} \Omega, 20 \mathrm{k} \Omega, 25 \mathrm{k} \Omega$ and $30 \mathrm{k} \Omega$. The corresponding amplitudes were $165.31 \mathrm{~nm}, 207.70 \mathrm{~nm}$, $317.63 \mathrm{~nm}$ and $483.04 \mathrm{~nm}$, respectively. Four grooves were cut with different magnitudes under the controlled amplitude condition. The experimental results acquired by AFM are shown in Fig. 16. Sectional views of these cut grooves are shown in Fig. 17. The results indicate that deeper groove cutting is achieved because of the greater magnitude of the controlled amplitude, as illustrated in Fig. 18. It is thus demonstrated experimentally that grooves can be cut with various depths under the $400 \mu \mathrm{N}$ pressing load with controlled amplitudes by varying the phase difference in the self-excitation cutting method.

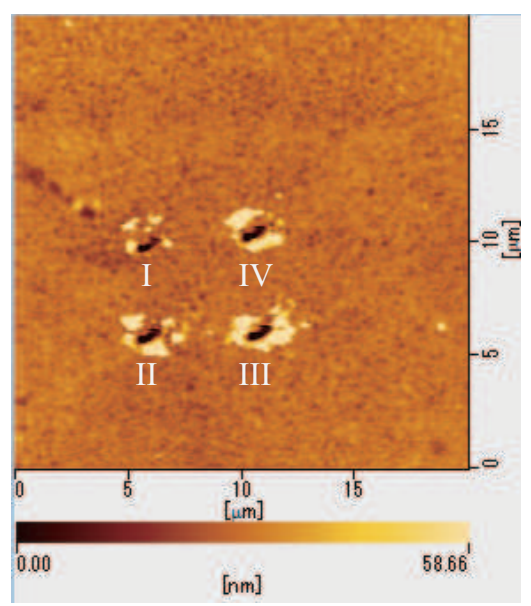

Fig. 16 Image of grooves cut under the $400 \mu \mathrm{N}$ pressing load by phase difference modulation with the self-excitation cutting method. Grooves I, II, III, and IV were cut while the resistance values were set at $15 \mathrm{k} \Omega$, $20 \mathrm{k} \Omega, 25 \mathrm{k} \Omega$ and $30 \mathrm{k} \Omega$, respectively.
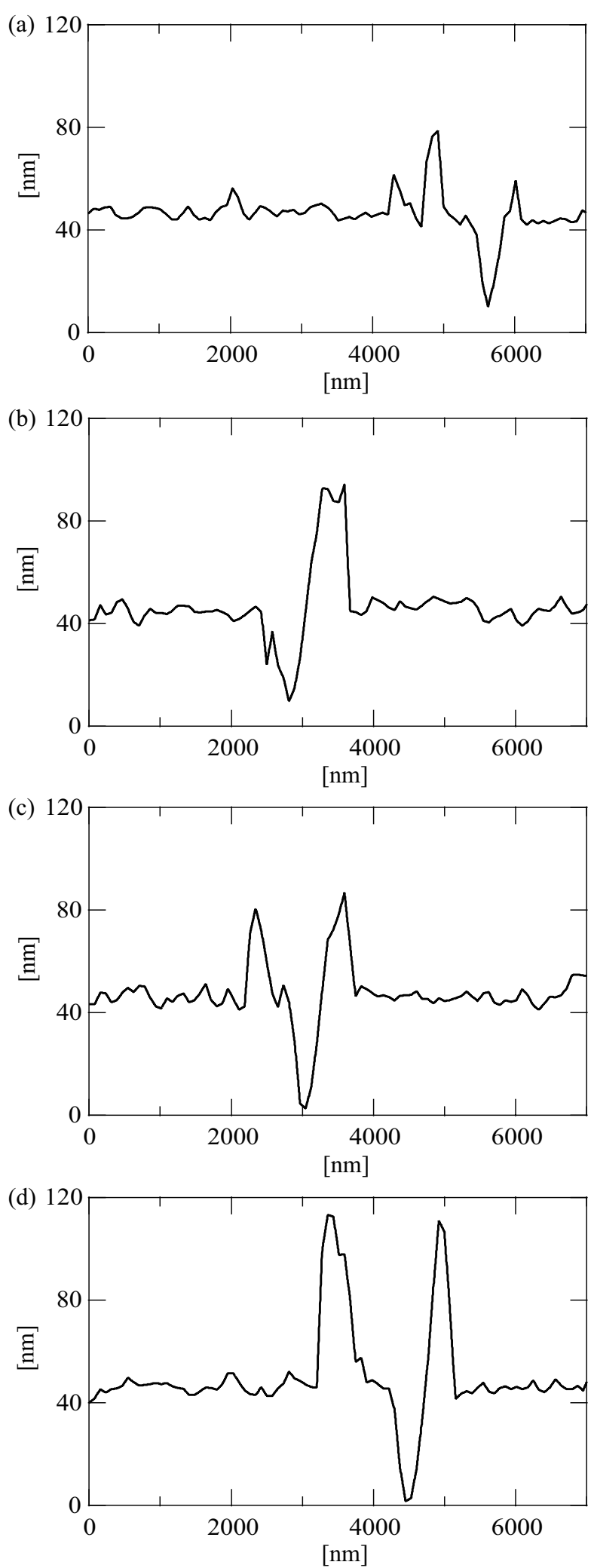

Fig. 17 Sectional views of four cut grooves. (a), (b), (c) and (d) represent the sectional profiles of the cut grooves I, II, III and IV, respectively. 


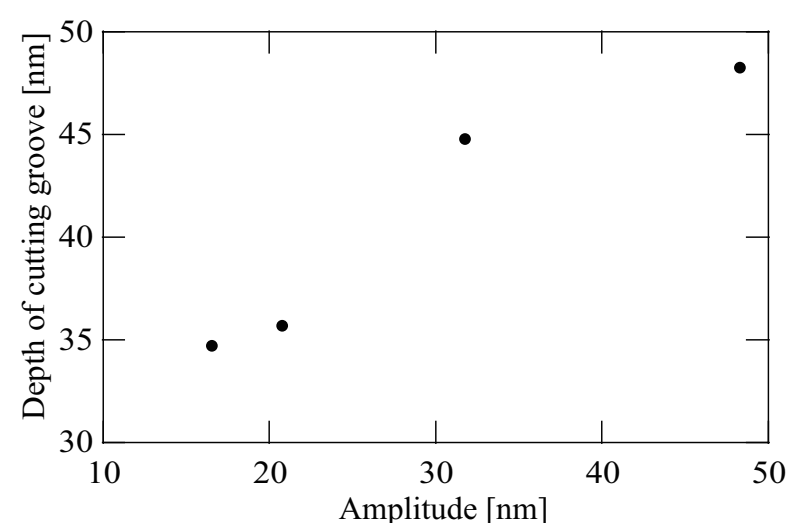

Fig. 18 Changes in amplitude when the microcantilever was selfexcited under the $400 \mu \mathrm{N}$ pressing load caused by changing the phase difference when the resistance values were $15 \mathrm{k} \Omega, 20 \mathrm{k} \Omega, 25 \mathrm{k} \Omega$ and $30 \mathrm{k} \Omega$.

\section{Conclusion}

A self-excitation cutting method is proposed to improve the efficiency of the nanoscale cutting. The validity and usefulness of the proposed method are demonstrated through a series of cutting experiments. Conclusions are as follows:

- The deeper grooves can be cut under application of the same pressing load than using the external excitation cutting method, because the microcantilever can be maintained in its resonant state independently of the pressing load when using the proposed method.

- The amplitude of the microcantilever has been demonstrated experimentally to be controlled to have different magnitudes by shifting the phase difference between the microcantilever deflection vibration signal and the feedback signal.

- The relationship between the amplitude and the groove cutting depth has been used to demonstrate that the deeper grooves are cut at larger magnitudes of the controlled amplitude.

\section{Acknowledgement}

This work was supported by Grant of "Jyu-ten-haibun" from University of Tsukuba.

\section{Compliance with ethical standards}

Conflict of interest: The authors declare that they have no conflict of interest.

\section{References}

1. Frazier, A.B., Warrington, R.O., Friedrich, C.: The miniaturization technologies: past, present, and future. IEEE Trans. Ind. Electron. 42, 423-430 (1995)

2. Kobayashi, D., Kim, C.-J., Fujita, H.: Photoresist-assisted release of movable microstructures. Jpn. J. Appl. Phys. 32, L1642-L1644 (1993)

3. Rangelow, I.W.: Dry etching-based silicon micro-machining for MEMS. Vacuum. 62, 279-291 (2001)

4. Williams, K.R., Muller, R.S.: Etch rates for micromachining processing. J. Microelectromech. Syst. 5, 256-269 (1996)

5. Williams, K.R., Gupta, K., Wasilik, M.: Etch rates for micromachining processing - part II. J. Microelectromechan. Syst. 12, 761778 (2003)

6. Xu, X.: Laser-based fabrication of polymer micropump. J. Micro Nanolithogr. MEMS MOEMS. 3, 152 (2004)

7. Reyntjens, S., Puers, S.: A review of focused ion beam applications in microsystem technology. J. Micromech. Microeng. 11, 287-300 (2001)

8. Banks, T.: Quantum Mechanics: An Introduction. CRC Press, Boca Raton (2019)

9. Orloff, J.: High-resolution focused ion-beams. Rev. Sci. Instrum. 64, 1105-1130 (1993)

10. Herrera-Granados, G., Ashida, K., Ogura, I., Okazaki, Y., Morita, N., Hidai, H., Matsusaka, S., Chiba, A.: Development of a non-rigid micro-scale cutting mechanism measuring the cutting force using an optical lever. Int. J. Autom. Technol. 8, 903-911 (2014)

11. Herrera-Granados, G., Morita, N., Hidai, H., Matsusaka, S., Chiba, A., Ashida, K., Ogura, I., Okazaki, Y.: Experimental stress analysis of glass cutting using a non-rigid cutting mechanism. Manuf. Lett. 6, 14-18 (2015)

12. Herrera-Granados, G., Morita, N., Hidai, H., Matsusaka, S., Chiba, A., Ashida, K., Ogura, I., Okazaki, Y.: Development of a nonrigid micro-scale cutting mechanism applying a normal cutting force control system. Precis. Eng. 43, 544-553 (2016)

13. Binnig, G., Quate, C.F., Gerber, C.: Atomic force microscope. Phys. Rev. Lett. 56, 930-933 (1986)

14. Ashida, K., Morita, N., Yoshida, Y.: Study on nano-machining process using mechanism of a friction force microscope. JSME Int. J. Series C. 44, 244-253 (2001)

15. Lysenko, O.G.: The use of scanning probe microscope with a diamond tip as a nanotechnological tool. J. Superh. Mater. 31, 387-391 (2009)

16. Miyake, S.: $1 \mathrm{~nm}$ deep mechanical processing of muscovite mica by atomic force microscopy. Appl. Phys. Lett. 67, 2925-2927 (1995)

17. Yongda, Y., Yanquan, G., Zhenjiang, Hu.: Recent advances in AFM tip-based nanomechanical machining. Int. J. Mach. Tools Manuf. 99, 1-18 (2015)

18. Shamoto, E., Moriwaki, T.: Study on elliptical vibration cutting. Manuf. Technol. 43, 35-38 (1994)

19. Park, S.S., Mostofa, M.G., Park, C.I., Mehrpouya, M., Kim, S.: Vibration assisted nano mechanical machining using afm probe. Manuf. Technol. 63, 537-540 (2014)

20. Kawasegi, N., Niwata, T., Morita, N., Nishimura, K., Sasaoka, H.: Improving machining performance of single-crystal diamond tools irradiated by a focused ion beam. Precis. Eng. 38, 174-182 (2014)

21. Roark, R.J., Young, W.C., Budynas, R.G.: Roark's formulas for stress and strain. McGraw-Hill, New York (2001)

22. Albrecht, T.R., Grutter, P., Horne, D., Rugar, D.: Frequency modulation detection using high-Q cantilevers for enhanced force microscope sensitivity. J. Appl. Phys. 69, 668-673 (1991)

23. Okajima, T., Sekiguchi, H., Arakawa, H., Ikai, A.: Self-oscillation technique for afm in liquids. Appl. Surf. Sci. 210 (1-2), 68-72 (2003)

24. Thompson, J.M.T., Stewart, H.B.: Nonlinear dynamics and chaos. Wiley (2002). 
25. Yabuno, H., Kuroda, M., Someya, T., Nishimura, K., Hayashi, K., Ashida, K.: Van der pol-type self-excited microcantilever probe for atomic force microscopy. Jpn. J. Appl. phys. 50, (2011):076601. 


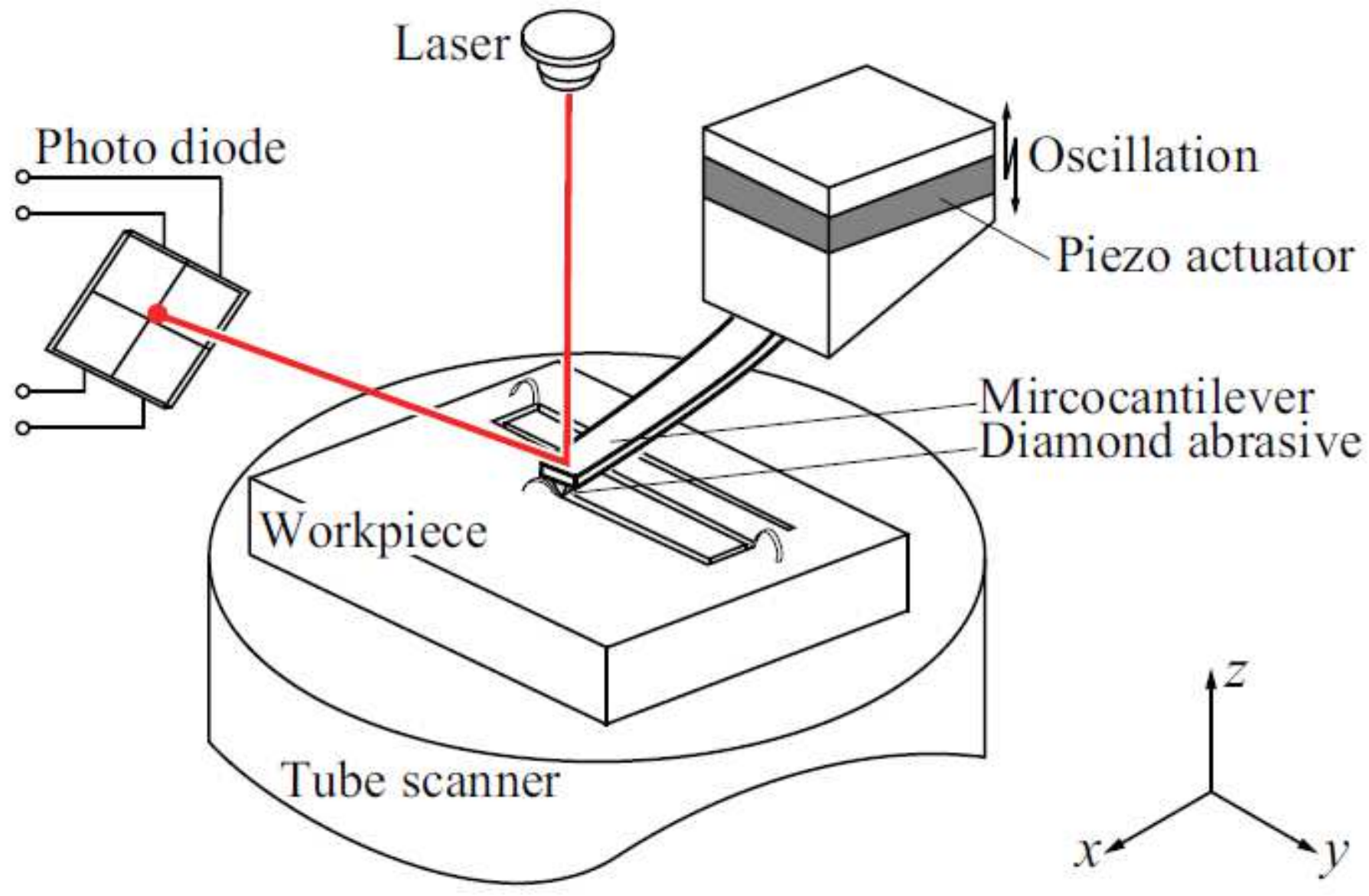

\section{Figure 1}

Schematic diagram of AFM system applied to nanoscale vibration cutting method. The system includes a workpiece, a microcantilever with a diamond set on its tip, a piezo actuator to enable the microcantilever oscillation, an optical angle sensor consisting of a laser and a quadrant photodiode to measure the deflection of the microcantilever tip, a tube scanner to move the workpiece in the $x-y$ plane and vary the distance between the microcantilever and the workpiece in the z-direction. 
(a)

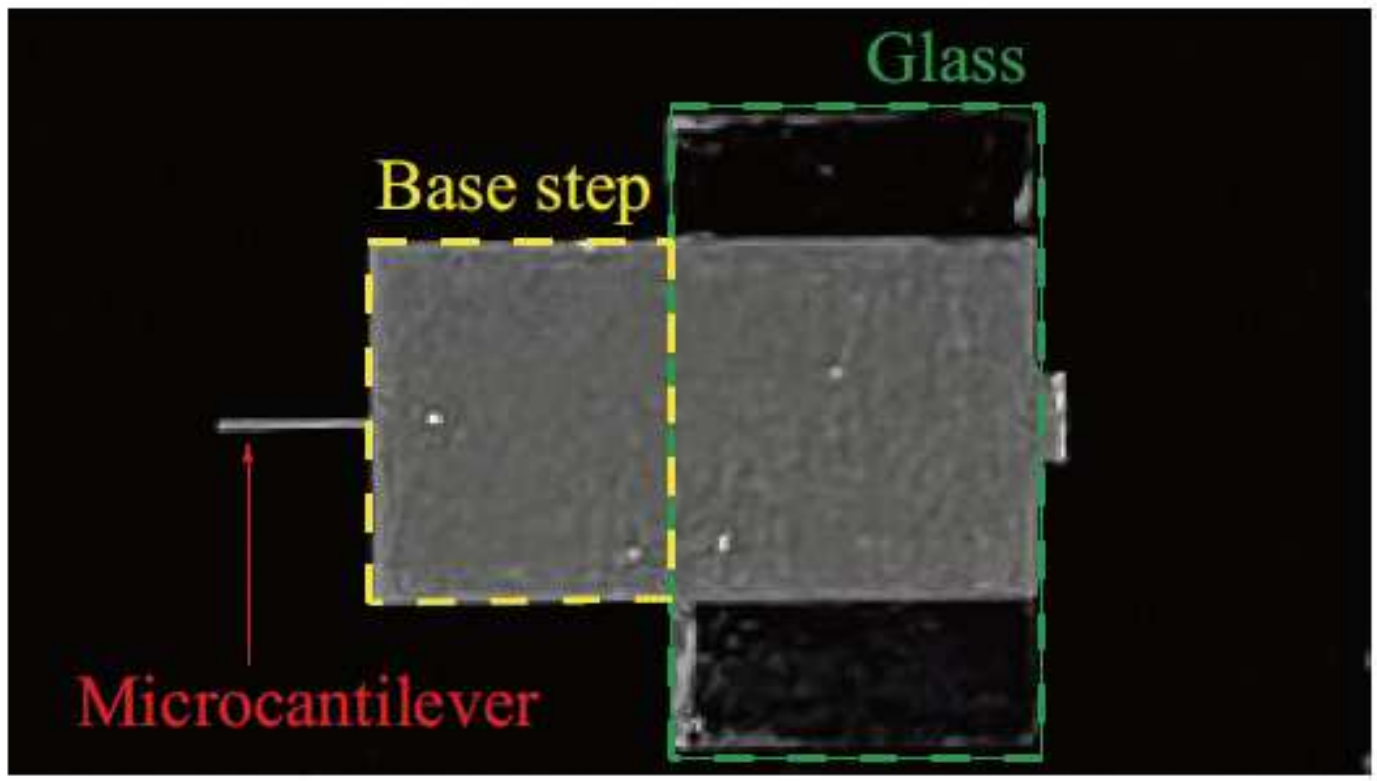

(b)

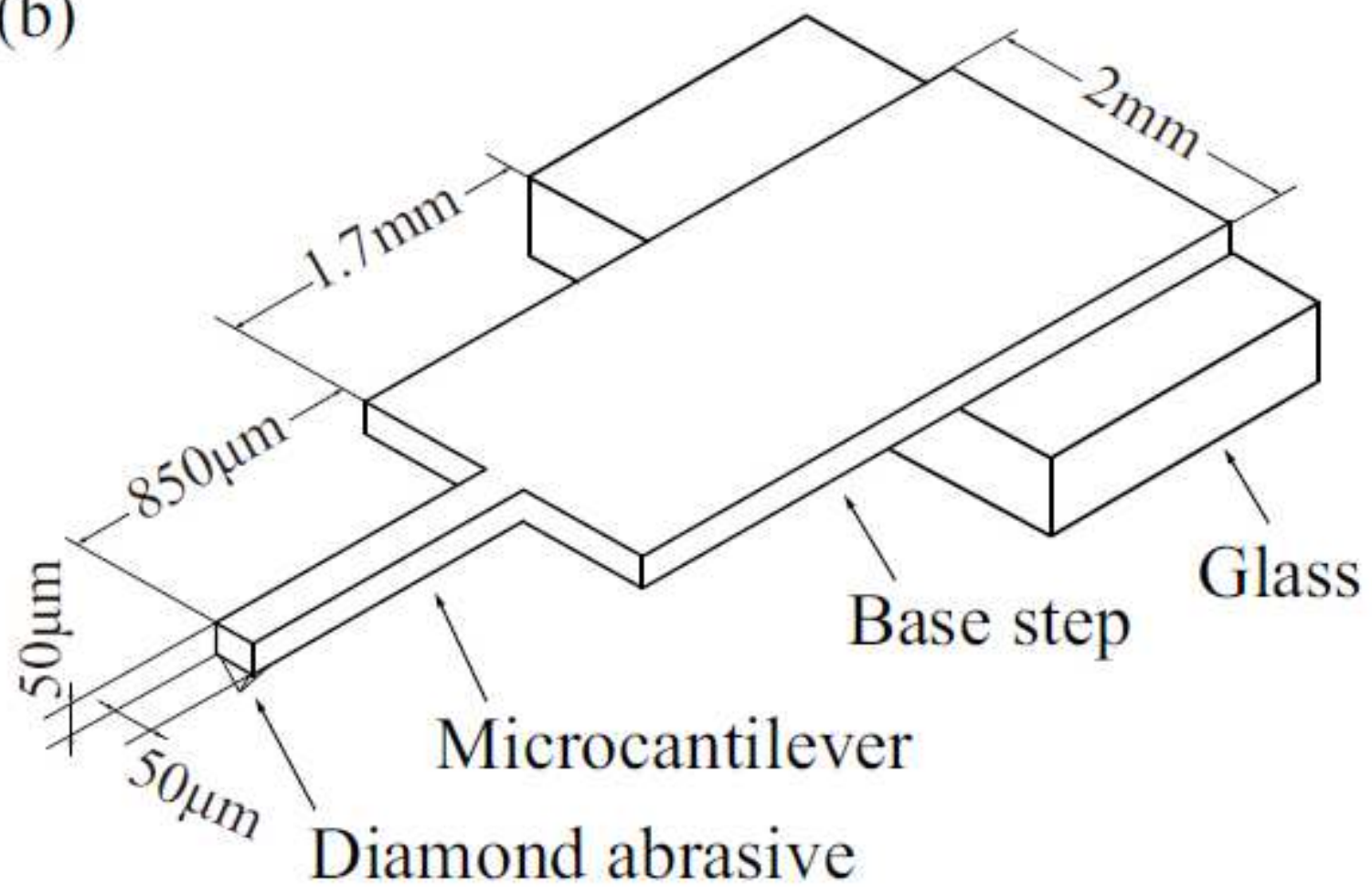

Figure 2

Photo (a) and Schematic diagram (b) of redesigned microcantilever. The length, width and thickness of the base step are $1.7 \mathrm{~mm}, 2 \mathrm{~mm}$ and $50 \mu \mathrm{m}$, respectively. The length, width and thickness of the microcantilever are $850 \mu \mathrm{m}, 50 \mu \mathrm{m}$ and $50 \mu \mathrm{m}$, respectively. 


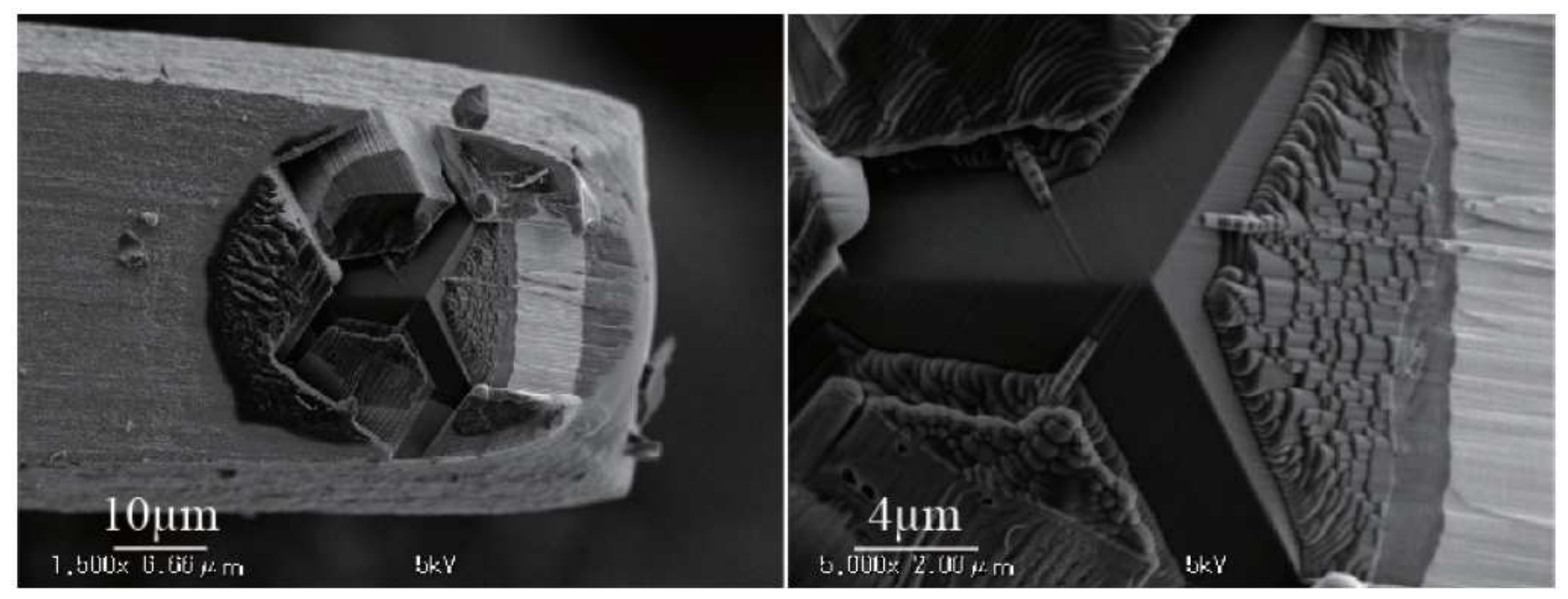

\section{Figure 3}

Diamond abrasive. This abrasive is set on the microcantilever tip and fabricated using focused ion beam technique. The angle of the fabricated diamond is 90 degrees; this angle is formed by the sides and the triangular faces at the profile vertex.

\section{Figure 4}

Response frequency curve (a) in air and (b) under a $400 \mu \mathrm{N}$ pressing load. The first natural frequencies of the microcantilever and the base step in air were experimentally measured to be $44.35 \mathrm{kHz}$ and 12.88 $\mathrm{kHz}$, respectively. The first natural frequency under a $400 \mu \mathrm{N}$ pressing load was experimentally measured to be $15.49 \mathrm{kHz}$. 


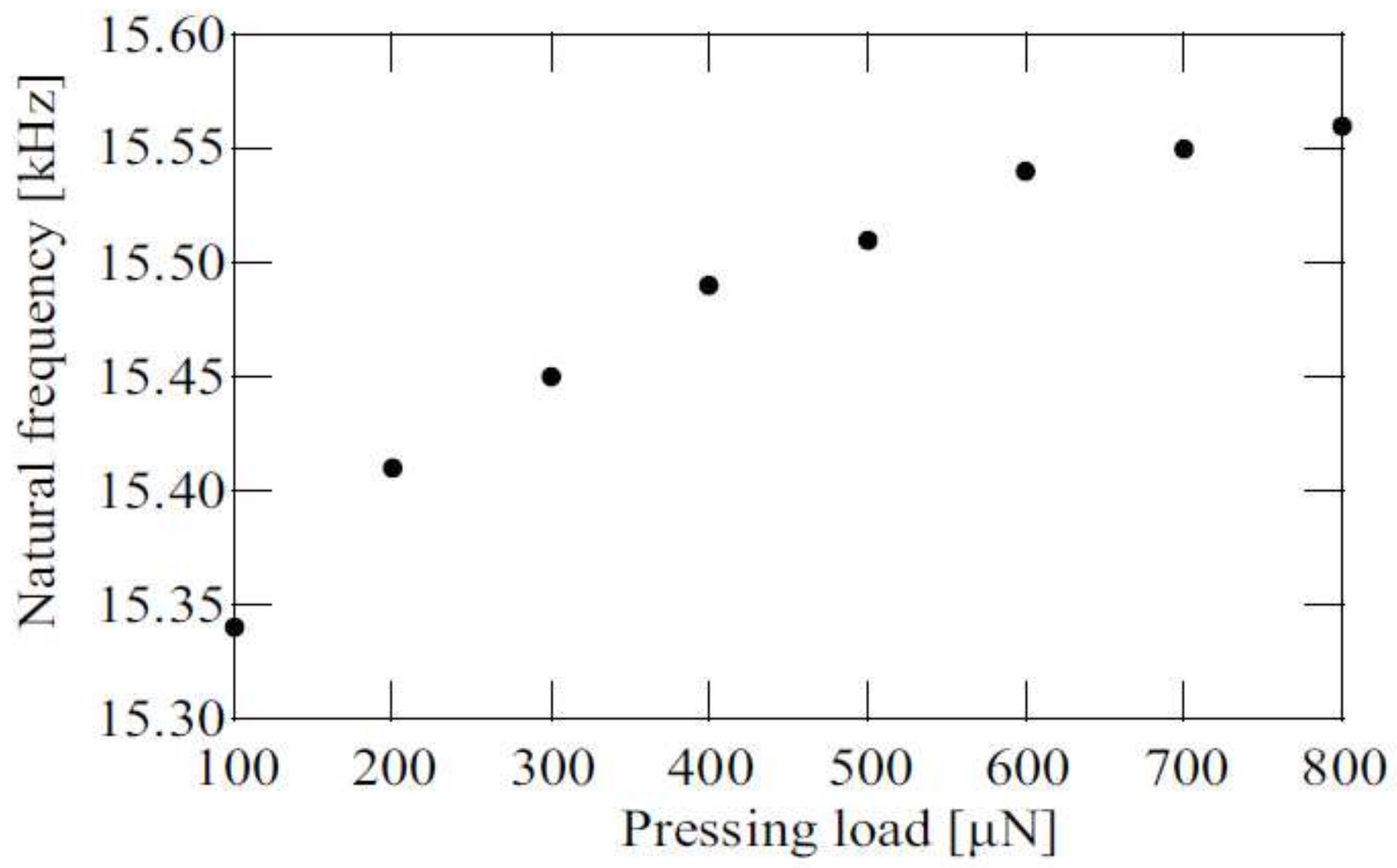

Figure 5

Natural frequency of microcantilever under various pressing loads with application of external excitation. 


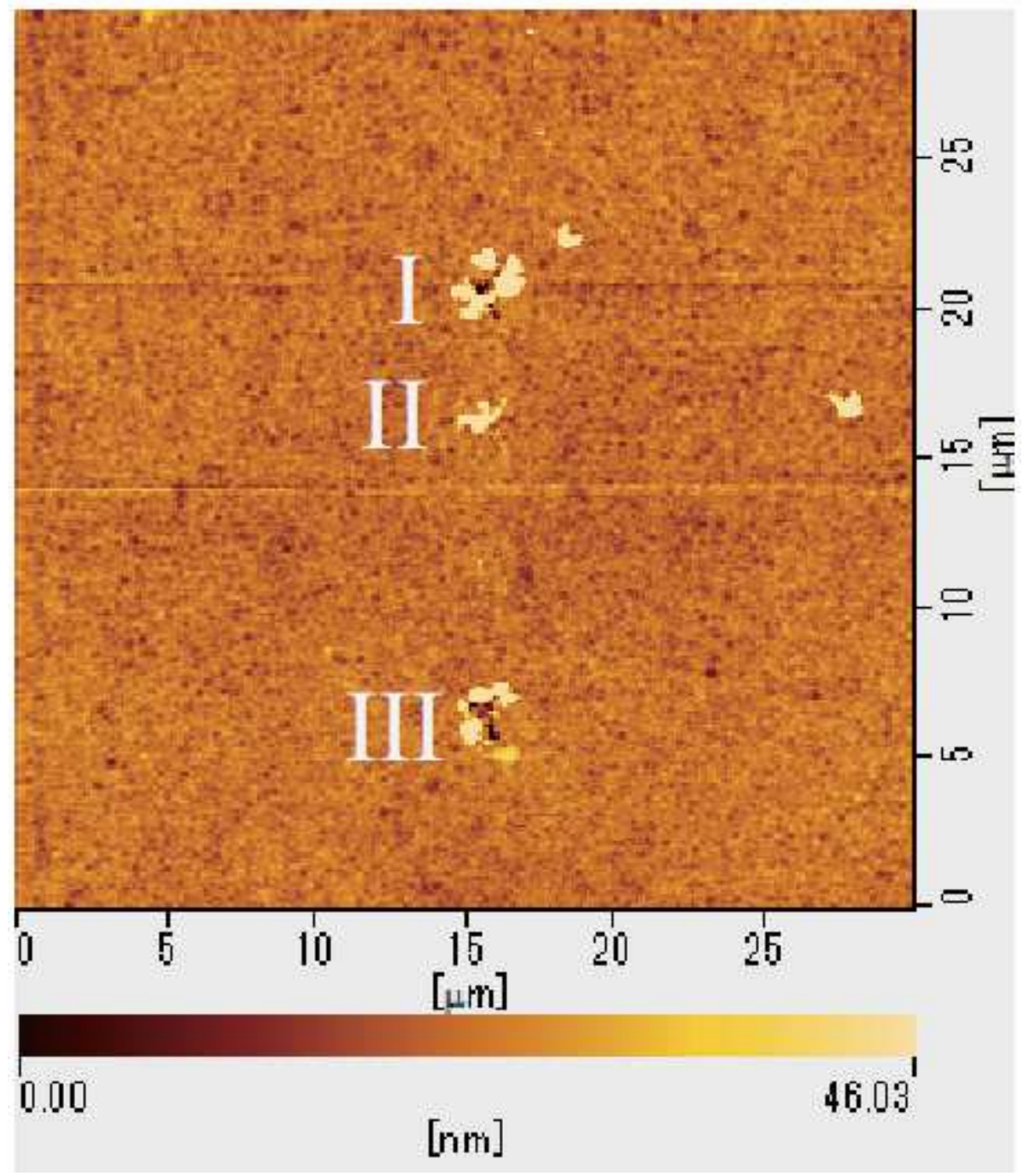

Figure 6

Image of grooves cut with the external excitation cutting method acquired using AFM. The upper groove is I, the middle groove is II and the lower groove is III. 

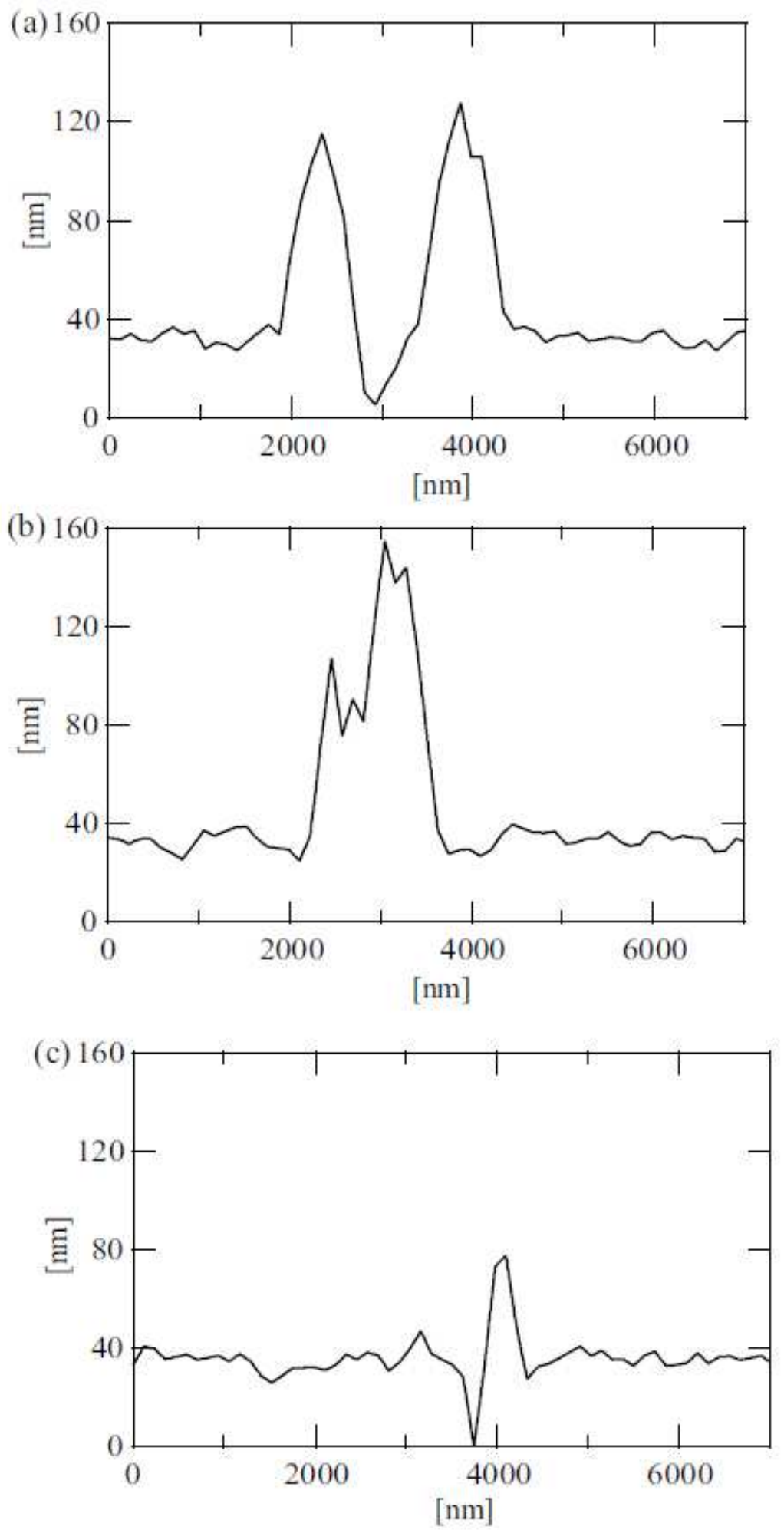

\section{Figure 7}

Sectional views of three cut grooves acquired using AFM. (a), (b) and (c) represent the sectional views of the three cut grooves I, II and III, respectively. 


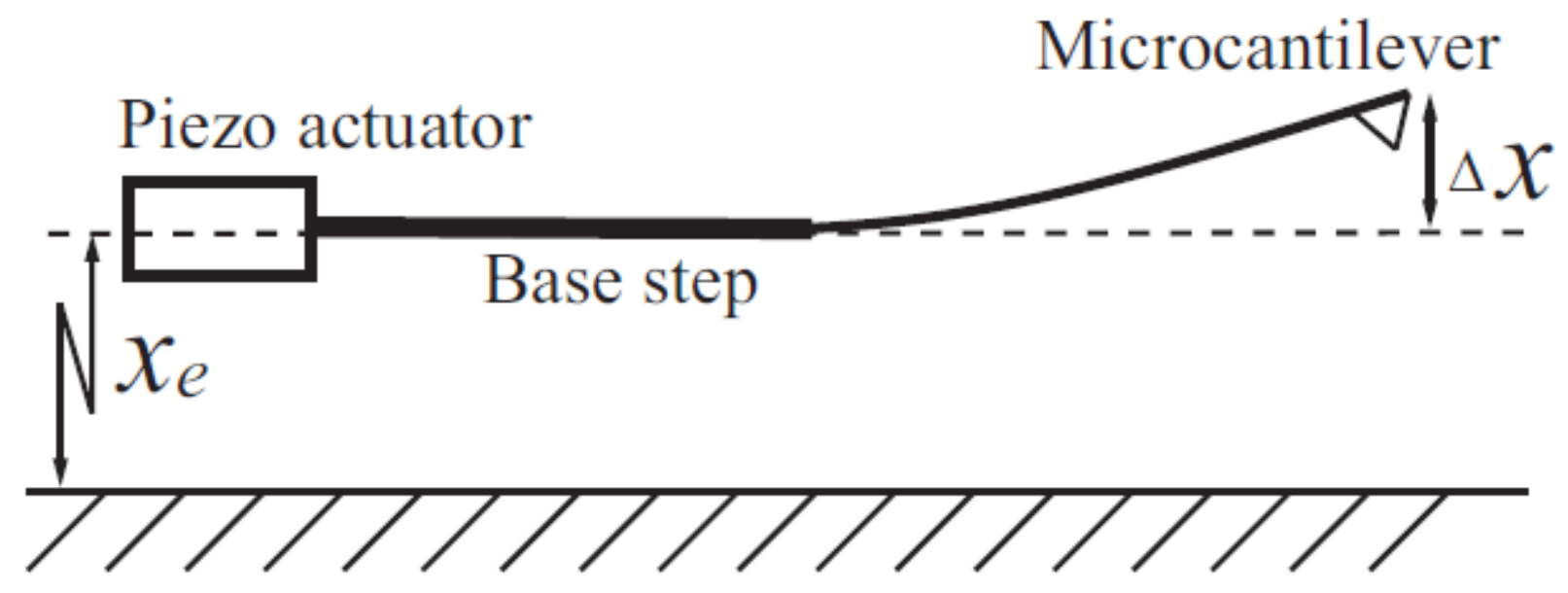

Figure 8

Schematic diagram of self-excited microcantilever. 
(a)

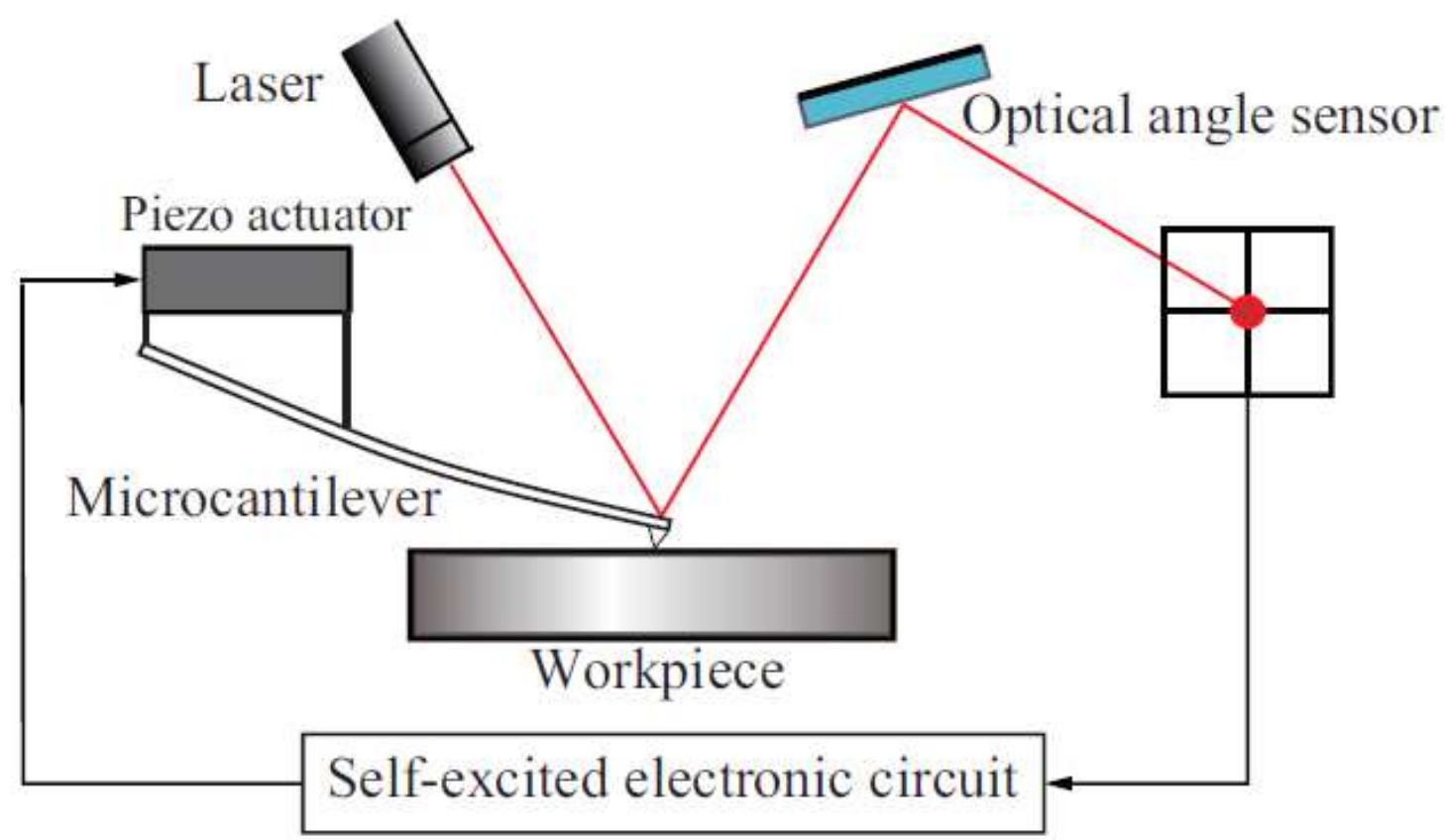

(b) Voltage follower Phase shifter Inverted amplifter (I)
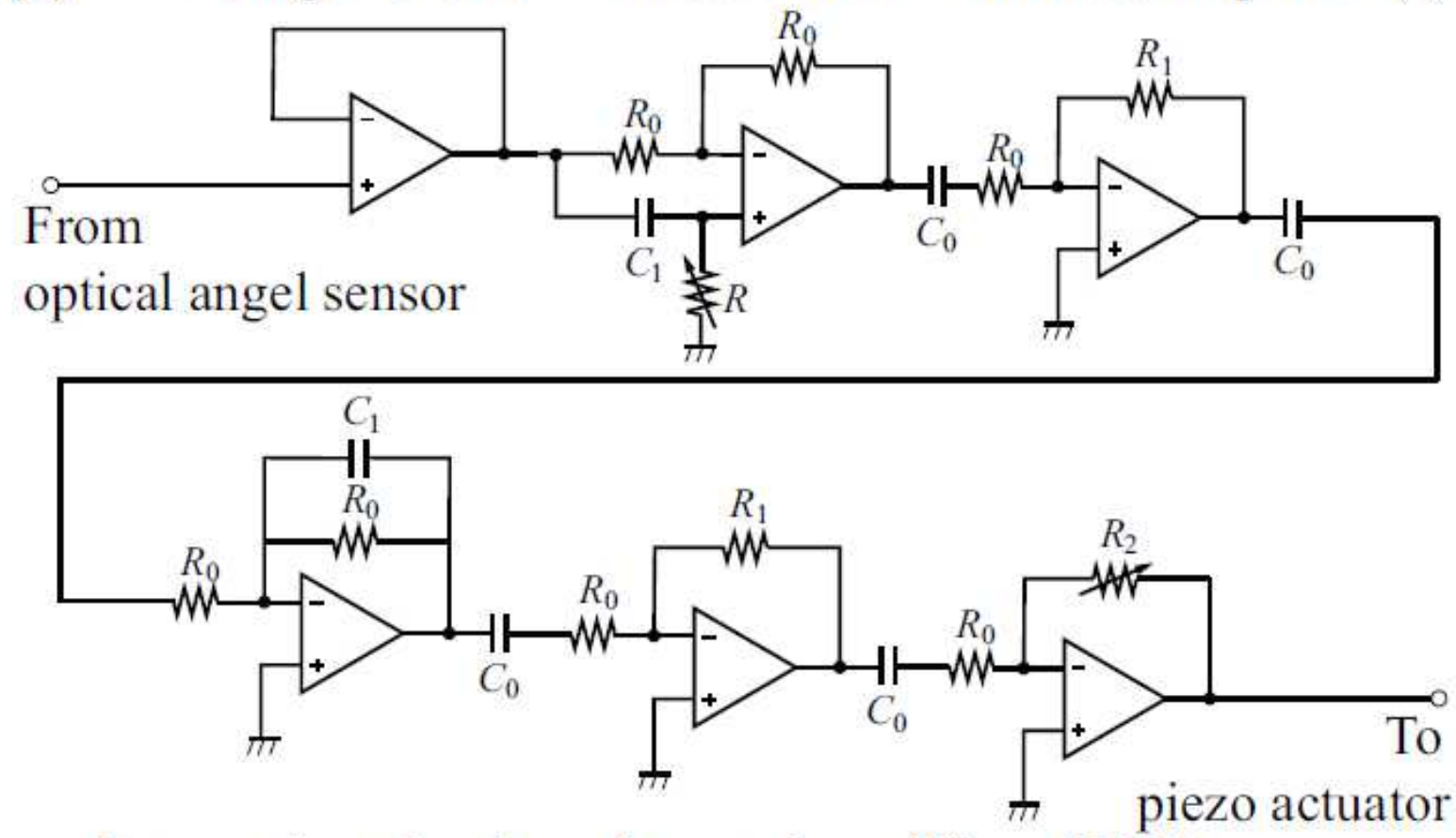

Intergrating circuit Inverted amplifters (II III)

$$
R_{0}=10 \mathrm{k} \Omega \quad R_{1}=100 \mathrm{k} \Omega \quad R_{2}=0 \sim 500 \mathrm{k} \Omega \quad C_{0}=1000 \mathrm{nF} \quad C_{1}=1000 \mathrm{pF}
$$

\section{Figure 9}

Schematic diagram of the self-excited system. (a) includes a workpiece, a microcantilever, a piezo actuator, a self-excited electronic circuit and an optical angle sensor that consists of a laser and a photodiode. (b) includes a voltage follower, a phase shifter, an integrating circuit and three inverting amplifiers. 


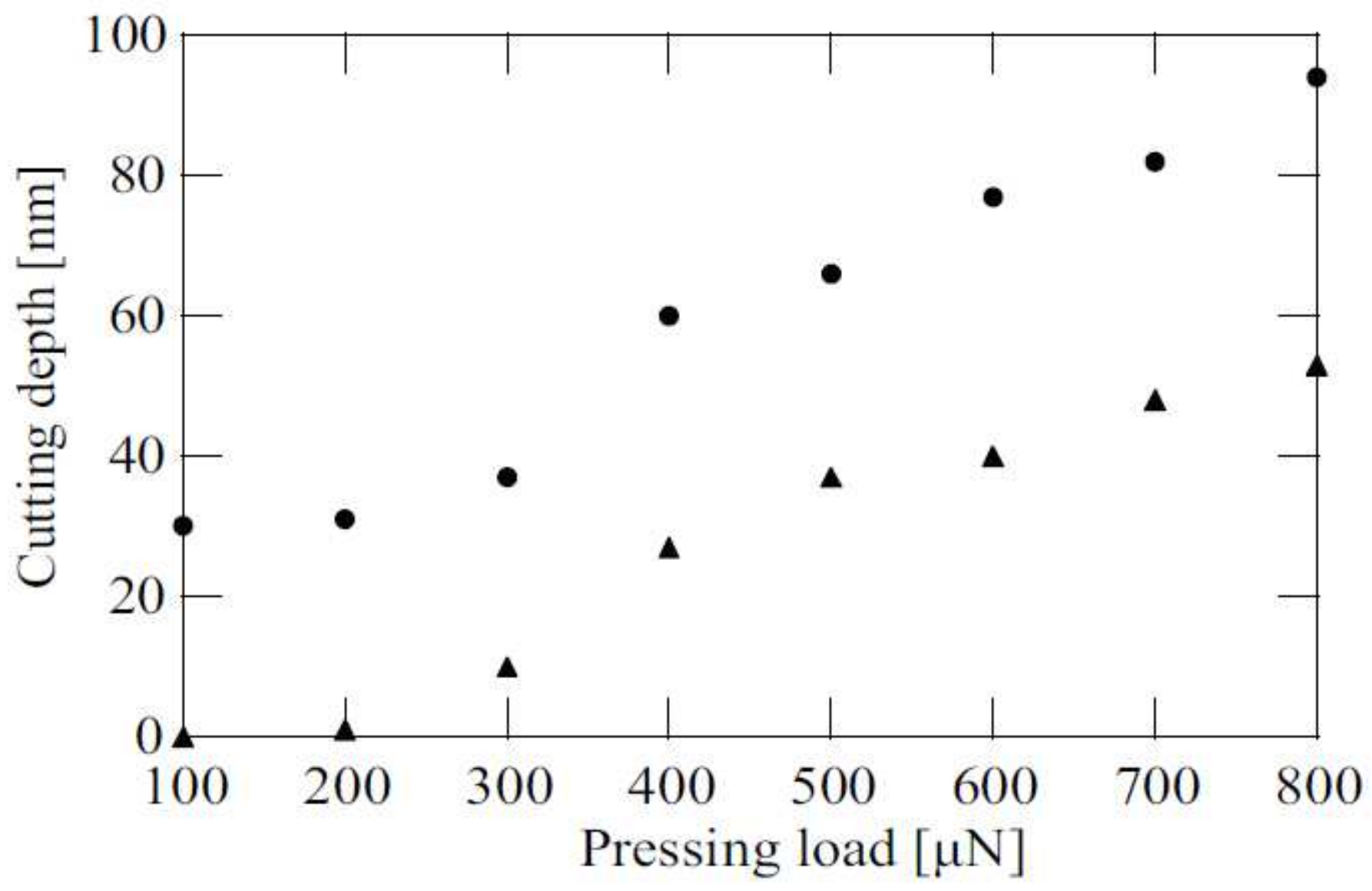

Figure 10

Depths of grooves cut under each pressing load with two types of cutting method. The triangles and the dots represent the depths using the external excitation cutting method and the self-excitation cutting method, respectively. 


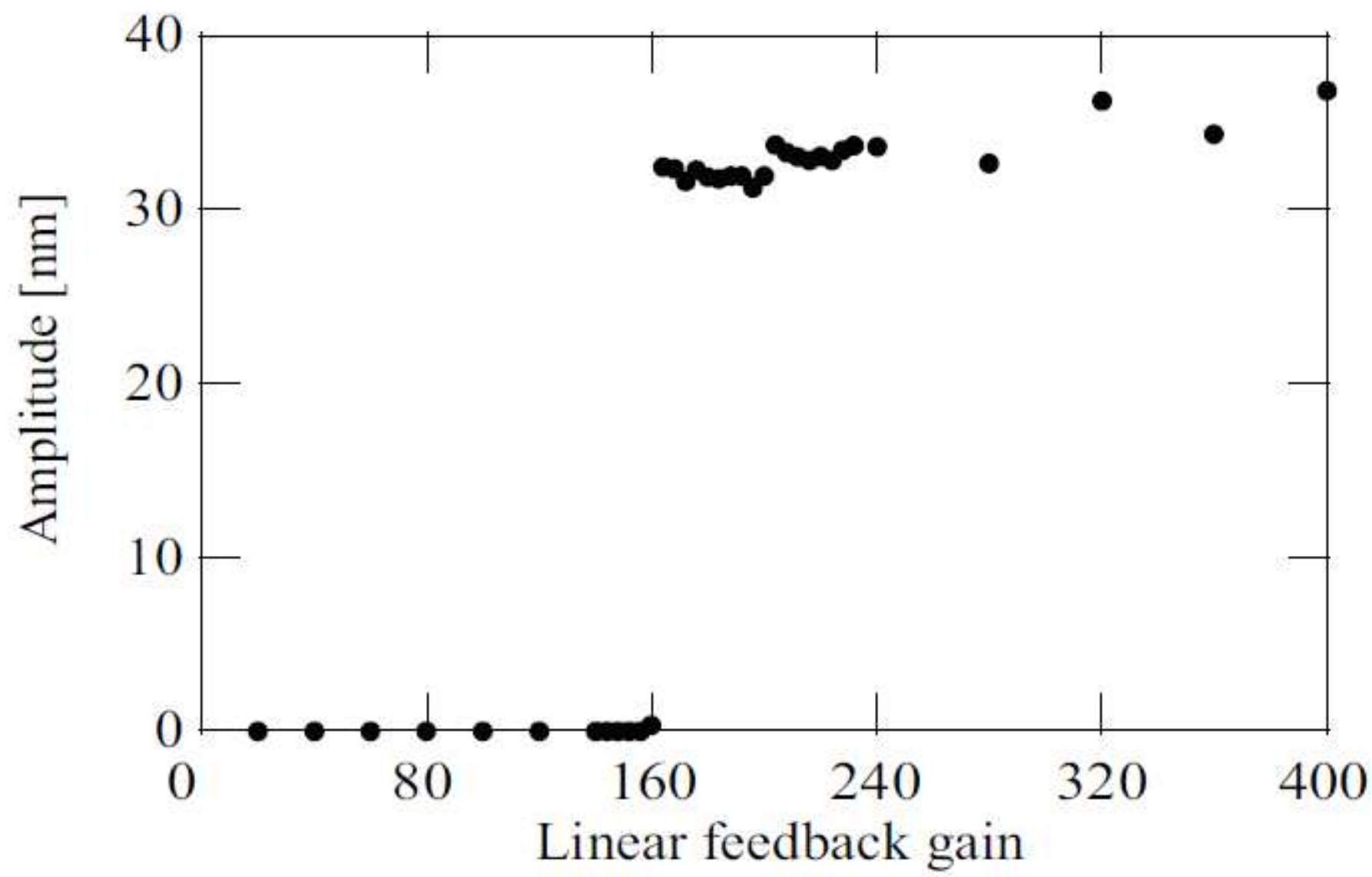

Figure 11

Change in amplitude caused by controlling linear feedback gain klin [1/s] under application of a $400 \mu \mathrm{N}$ pressing load. 


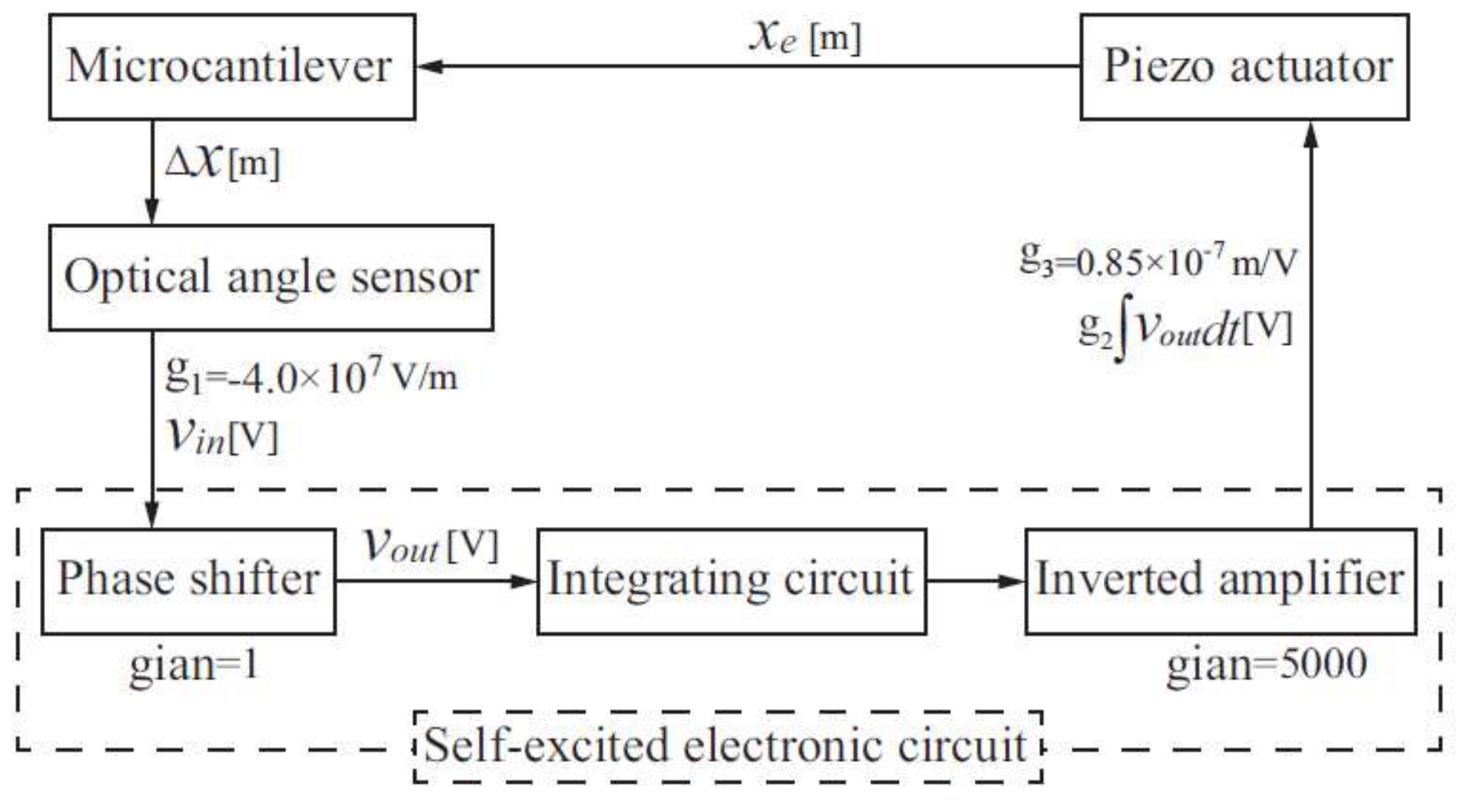

Figure 12

Block diagram of the self-excited system. 


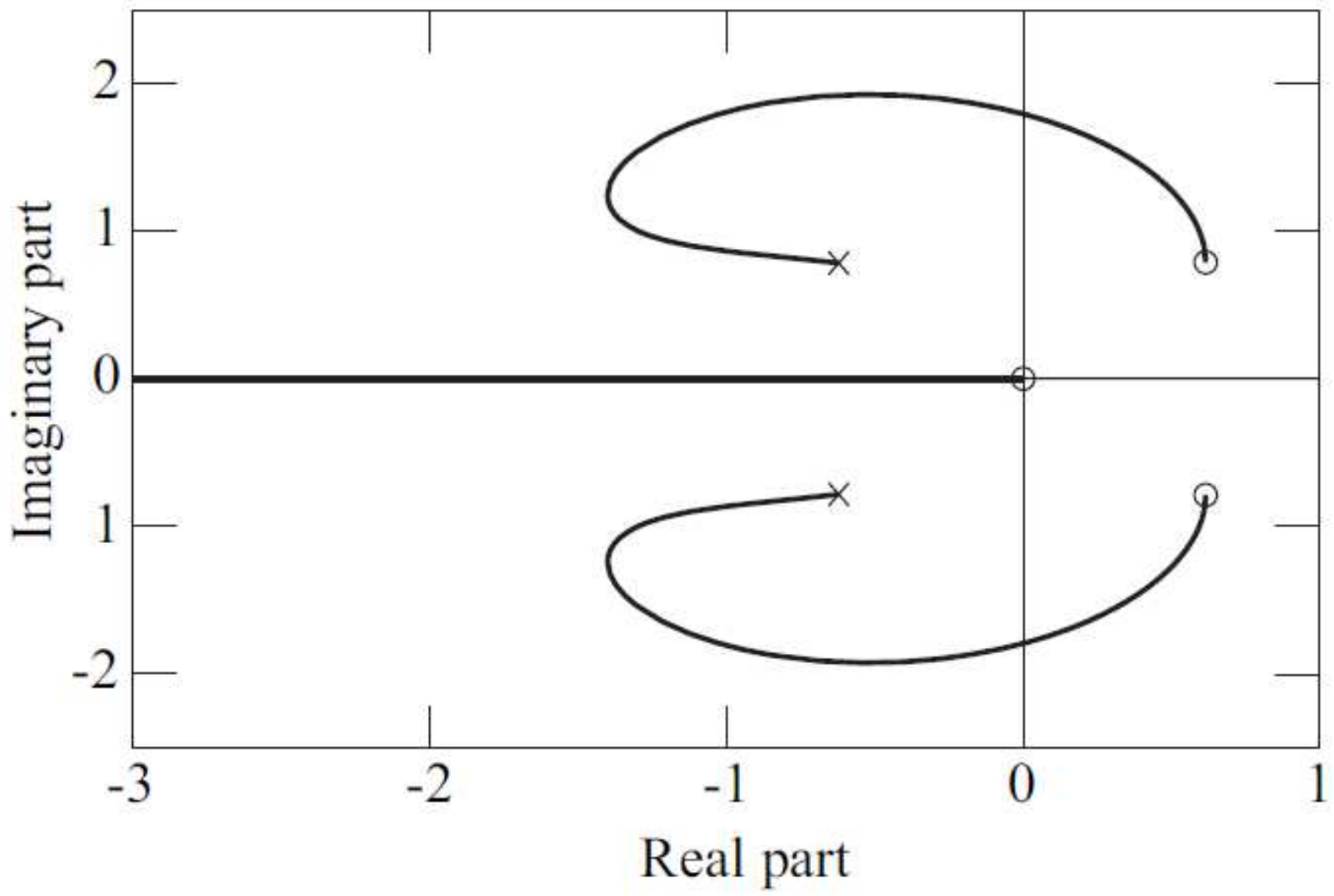

Figure 13

Root locus of the self-excited system. 

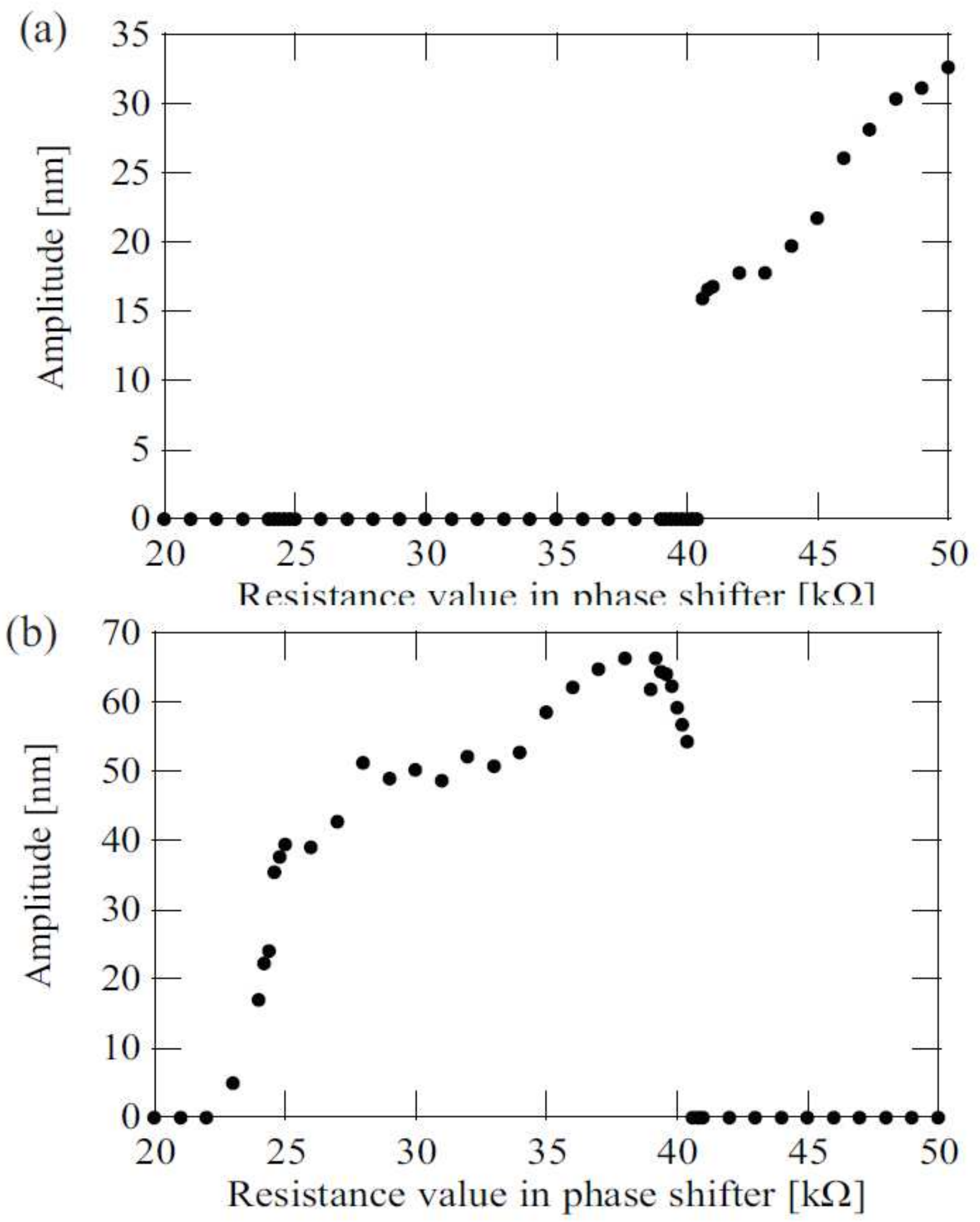

Figure 14

Change in the amplitude magnitude in air caused by phase modulation. (a) and (b) show the amplitude magnitude changes in the cases when the base step and the microcantilever are self-excited, respectively, while the resistance value in the phase shifter is varied. 

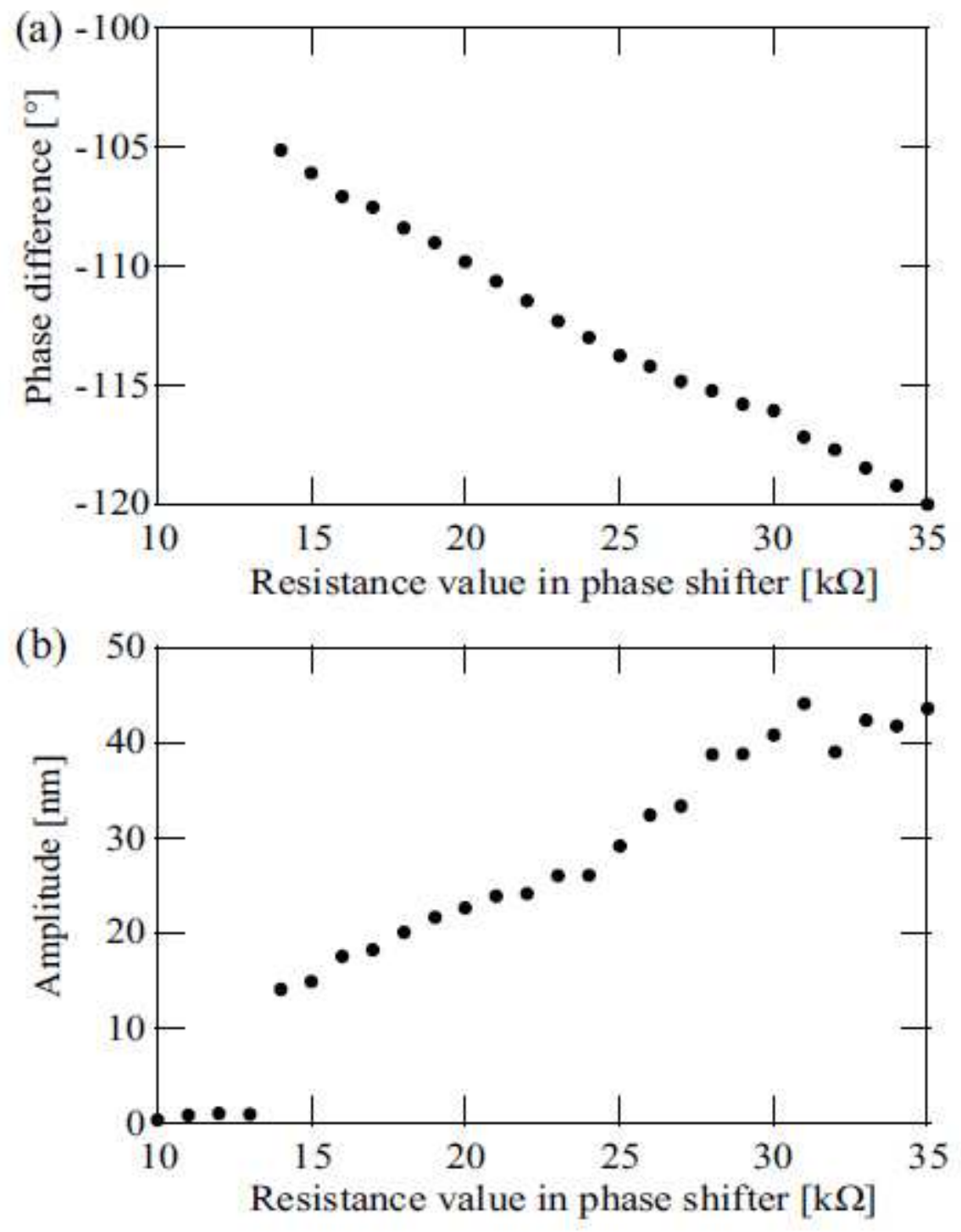

Figure 15

Results for the phase difference and amplitude changes caused by phase modulation under application of a $400 \mu \mathrm{N}$ pressing load while the resistance value in the phase shifter was varied from $10 \mathrm{k} \Omega$ to $35 \mathrm{k} \Omega$. (a) Change in the phase difference. (b) Change in the amplitude magnitude.

\section{Figure 16}

Image of grooves cut under the $400 \mu \mathrm{N}$ pressing load by phase difference modulation with the selfexcitation cutting method. Grooves I, II, III, and IV were cut while the resistance values were set at $15 \mathrm{k} \Omega$, $20 \mathrm{k} \Omega, 25 \mathrm{k} \Omega$ and $30 \mathrm{k} \Omega$, respectively. 

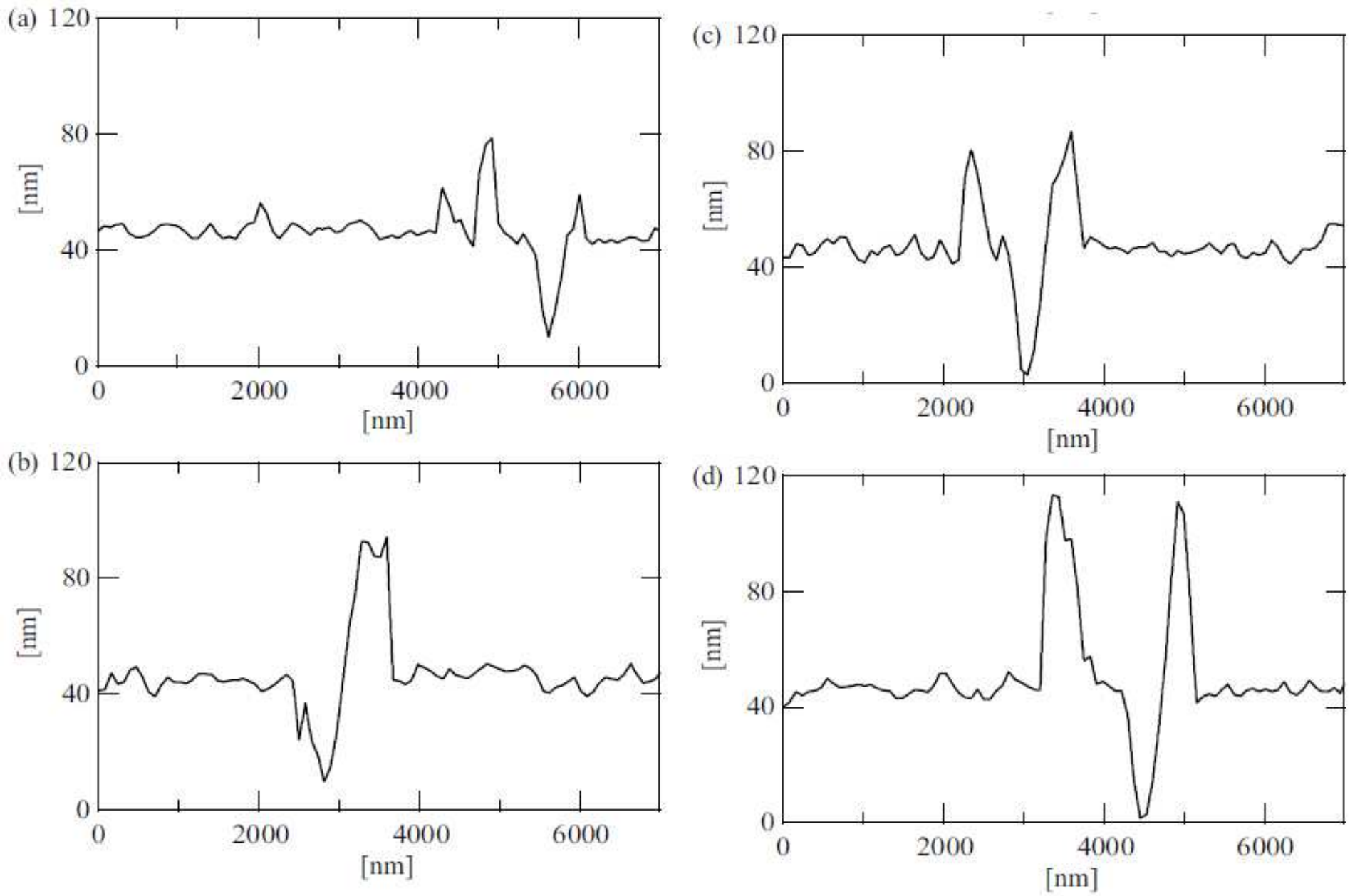

Figure 17

Sectional views of four cut grooves. (a), (b), (c) and (d) represent the sectional profiles of the cut grooves I, II, III and IV, respectively.

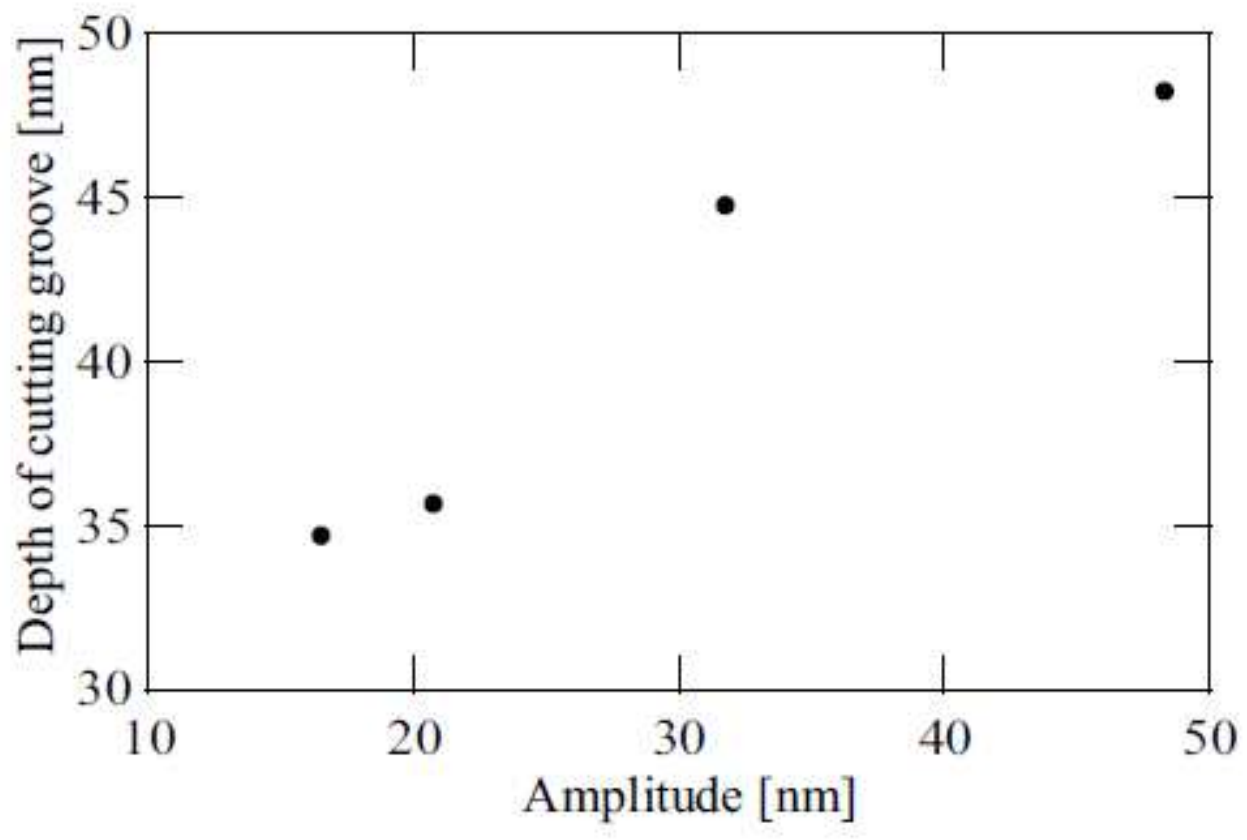


Figure 18

Changes in amplitude when the microcantilever was selfexcited under the $400 \mu \mathrm{N}$ pressing load caused by changing the phase difference when the resistance values were $15 \mathrm{k} \Omega, 20 \mathrm{k} \Omega, 25 \mathrm{k} \Omega$ and $30 \mathrm{k} \Omega$. 